\title{
Pacific
}

Journal of

Mathematics

\section{ON SLOPE GENERA OF KNOTTED TORI IN 4-SPACE}

Yi LiU, Yi Ni, Hongbin SUn AND Shicheng WANG 


\title{
ON SLOPE GENERA OF KNOTTED TORI IN 4-SPACE
}

\author{
Yi LiU, Yi Ni, HoNGBin SUN AND SHICHENG WANG
}

\begin{abstract}
We investigate genera of slopes of a knotted torus in the 4-sphere analogous to the genus of a classical knot. We compare various formulations of this notion, and use this notion to study the extendable subgroup of the mapping class group of a knotted torus.
\end{abstract}

1. Introduction 117

2. Background 119

3. Genera of slopes 121

4. Induced seminorms on $H_{1}\left(T^{2} ; \mathbb{R}\right) \quad 124$

5. Braid satellites 132

6. Miscellaneous examples 139

7. Further questions 141

Acknowledgements 141

References 142

\section{Introduction}

In classical knot theory, the genus of a knot in the 3-sphere is a basic numerical invariant which has been well-studied. In this note, we investigate some analogous notions for the slopes of a knotted torus in the 4-sphere $S^{4}$. These reflect certain essential differences between knotted tori and knotted spheres. Similar phenomena arise in the case of knotted surfaces in $S^{4}$, but the discussion would require more general treatments. We focus on the torus case in this note for the sake of simplicity.

A knotted torus in $S^{4}$ is a locally flat subsurface homeomorphic to the torus. Without loss of generality, we may fix a choice of marking (see Section 2B). Throughout this note, a knotted torus in $S^{4}$ means a locally flat embedding

$$
K: T^{2} \hookrightarrow S^{4}
$$

The second author was partially supported by an AIM Five-Year Fellowship and NSF grant numbers DMS-1021956 and DMS-1103976. The fourth author was partially supported by grant No.10631060 of the National Natural Science Foundation of China.

MSC2010: primary 57Q45; secondary 20F12.

Keywords: knotted surface, genus, extendable subgroup. 
from the torus to the 4-sphere. By slightly abusing the notation, we often write the image of $K$ still as $K$. For any slope (that is, an essential simple closed curve) $c \subset K$, it makes sense to define the genus

$$
g_{K}(c)
$$

of $c$ as the smallest possible genus of all the locally flat, orientable, compact subsurfaces $F \hookrightarrow S^{4}$ whose image bounds $c$ and meets $K$ exactly in $c$. The genus of a slope is clearly an isotopy invariant of the knotted torus, and indeed, it is invariant under extendable automorphisms. More precisely, if $\tau$ is an automorphism (that is, an orientation-preserving self-homeomorphism up to isotopy) of $T^{2}$ that can be extended over $S^{4}$ as an orientation-preserving self-homeomorphism, then $c$ and $\tau(c)$ must have the same genus for any slope $c \subset K$. It is clear that all such automorphisms form a subgroup

$$
\mathscr{E}_{K} \leq \operatorname{Mod}\left(T^{2}\right)
$$

of the mapping class group $\operatorname{Mod}\left(T^{2}\right)$, called the extendable subgroup with respect to $K$. See Section 3 for more details. A primary motivation of our study is to understand $\mathscr{E}_{K}$ with the aid of the slope genera.

Natural as it is, the genus of a slope of a knotted torus is usually hard to capture. In contrast, two weaker notions yield much more interesting applications. One of them is called the singular genus of a slope $c$, denoted $g_{K}^{\star}(c)$. It is defined by loosening the locally flat embedding condition on the bounding surface $F$ above, only requiring $F \rightarrow S^{4}$ to be continuous. Another is called the induced seminorm on $H_{1}\left(T^{2}\right)$, denoted $\|\cdot\|_{K}$. This is an analogue to the (singular) Thurston norm in the classical context. In Section 4, we prove an inequality relating the seminorms associated with the satellite construction, which is analogous to the classical Schubert inequality for knots in $S^{3}$.

A simple observation at this point is that both the singular genus and the seminorm of a slope are group-theoretic notions, which can be rephrased in terms of the commutator length and the stable commutator length in the fundamental group of the exterior of the knotted torus, respectively (Remarks 3.3, 4.5).

As an application of these results, we study braid satellites in Section 5. In particular, this allows us to obtain examples of knotted tori with finite extendable subgroups. In Section 6, we exhibit examples where the singular genus is positive for a slope with vanishing seminorm. This implies the singular genus is strictly stronger than the seminorm as an invariant associated to slopes. We also relate the vanishing of the singular genus for a slope $c \subset K$ to the extendability of the Dehn twist $\tau_{c} \in \operatorname{Mod}\left(T^{2}\right)$ along $c$ in a stable sense (Lemma 6.2).

Section 2 surveys results relevant to our discussion. A few questions for further study related to slope genera and the extendable subgroups are raised in Section 7. 


\section{Background}

This section briefly surveys the history relevant to our topic in several aspects. We hope that it will supply the reader some context for our discussion. However, the reader may safely skip this part for the moment, and perhaps come back later for further references. We thank the referee for suggesting us to include some of these materials.

2A. Genera of knots. For a classical knot $k$ in $S^{3}$, one of the most important numerical invariants is its genus $g(k)$, introduced by Herbert Seifert [1935]. It is naturally defined as the smallest genus among that of all possible Seifert surfaces of $k$; recall that a Seifert surface of $k$ is an embedded compact connected surfaces in $S^{3}$ whose boundary is $k$. In other words, if $k$ is not the unknot, the smallest possible complexity of a Seifert surface is $2 g(k)-1>0$.

In 3-dimensional topology, a suitable generalization of this notion for any orientable compact 3-manifold $M$ is the Thurston norm. It was introduced by William Thurston [1986]. Thurston discovered that the smallest possible complexity of properly embedded surface representatives for elements of $H_{2}(M, \partial M ; \mathbb{Z})$ can be linearly continuously extended over $H_{2}(M, \partial M ; \mathbb{R})$ to be a seminorm. It is actually a norm in certain cases, for example, if $M$ is hyperbolic of finite volume. Thurston then asked if this notion coincides with the one defined similarly using properly immersed surfaces, which was later known as the singular Thurston norm. The question was answered affirmatively by David Gabai [1983] using his sutured manifold hierarchy. As an immediate consequence, it was made clear that there is only one notion of genus (or complexity) for classical knots, whether we consider connected or disconnected, properly immersed or embedded Seifert surfaces.

Generally speaking, the genus of a knot is quite accessible. For a $(p, q)$-torus knot, where $p, q$ are coprime positive integers, the genus is well known to be $(p-1)(q-1) / 2$. For a satellite knot, the Schubert inequality yields a lower bound $\left(\hat{g}_{\mathrm{p}}+|w| \cdot g_{\mathrm{c}}\right)$ of the genus in terms of the genus $g_{\mathrm{c}}$ of the companion knot, the genus $\hat{g}_{\mathrm{p}}$ of the desatellite knot, and the winding number $w$ of the pattern [Schubert 1953]. Furthermore, the genus of a knot is known to be algorithmically decidable [Schubert 1961]. In fact, certifying an upper bound is NP-complete [Agol et al. 2006]. The genus can also be bounded and detected in terms of other more powerful algebraic invariants, such as the knot Floer homology [Ozsváth and Szabó 2004] and twisted Alexander polynomials [Friedl and Vidussi 2012].

2B. Knotting and marking. One of the classical problems in topology is the knotting problem, namely, "Are two embeddings of a given space into $n$-space isotopic?" Usually, the given space is a connected closed $m$-manifold $M$ where $m<n$, the embedding is locally flat, and the question can be made precise most naturally in 
the piecewise-linear or the smooth category. When the codimension is high enough, for example, if $n=2 m+1$ and $m>1$, all embeddings are isotopic to one another so they "unknot" in this sense [Wu 1958]. However, below the stable range, the knotting problem becomes very interesting, as we have already seen in the classical knot case.

Regarding an embedding of $M^{m}$ into $\mathbb{R}^{n}$ as a marking of its image, the knotting problem may be phrased to identify or distinguish knotting types (that is, isotopy classes) of marked submanifolds. Somewhat more naturally, one can ask if two unmarked knotted submanifolds are isotopic to each other, or precisely, if two embeddings are isotopic up to precomposing with an automorphism of $M$ in the given category. Suppose we have already solved the knotting problem. Then, the latter question amounts to asking whether two markings differ only by an extendable automorphism; see [Ding et al. 2012, Lemma 2.5]. Therefore, marking does not make a difference if $M$ has a trivial mapping class group in the category, for example, in the cases of classical knots and 2-knots, but it does in general if the extendable subgroup is a proper subgroup of the mapping class group; see [Ding et al. 2012; Hirose 1993; 2002; Montesinos 1983].

We refer the reader to the survey [Skopenkov 2008] for the embedding problem and the knotting problem in general dimensions.

2C. Knotted surfaces. The study of knotted surfaces can considered to be the middimensional knot theory. In this transitional zone between the low-dimensional case and the high-dimensional (2-codimensional) case, we find geometric-topological and algebraic-topological methods to have an interesting interaction. For extensive references on this topic, see the books [Kawauchi 1996; Hillman 1989; Carter and Saito 1998; Carter et al. 2004; Kamada 2002].

With an auxiliary choice of marking, let us write a knotted surface as a locally flat embedding $K: F \hookrightarrow \mathbb{R}^{4}$, where $F$ is a closed surface. We can visualize a knotted surface by drawing a diagram obtained via a generic projection of $K$ onto a 3-subspace, or by displaying a motion picture of links in $\mathbb{R}^{3}$, obtained via a generic line projection that is Morse when restricted to $K$; see [Carter and Saito 1998; Kawauchi et al. 1982]. The fundamental group of the exterior is called the knot group of $K$, denoted as $\pi_{K}$. Similar to the classical case, $\pi_{K}$ has a Wirtinger-type presentation in terms of its diagram [Yajima 1962], and $\pi_{K}$ can be isomorphically characterized by having an Artin-type presentation, described in terms of 2-dimensional braids [Kamada 2002].

Exteriors of knotted surfaces form an interesting family of 4-manifolds. The fundamental group of any such manifold is nontrivial, and it contains much information about the topology. For instance, it has been suspected for orientable knotted surfaces that having an infinite cyclic knot group implies unknotting, namely, that $K$ bounds an embedded handlebody [Hosokawa and Kawauchi 1979]. By deep 
methods of 4-manifold topology, this has been confirmed for knotted spheres in the topological category [Freedman and Quinn 1990, Theorem 11.7A]. In earlier studies of knotted surfaces, researchers frequently looked for examples with prescribed properties of the knot group, such as required deficiency [Fox 1962; Levine 1978; Kanenobu 1983], or required second homology [Brunner et al. 1982; Gordon 1981; Litherland 1981; Maeda 1977]. In some other constructions of particular topological significance, combinatorial group theory again plays an important role in verification [Gordon 1976; Kamada 1990; Livingston 1985; 1988].

Many of these constructions implement satellite knotting on various stages. The idea of such an operation is to replace a so-called companion knotted surface with another one that is embedded in the regular neighborhood of the former, often in a more complicated pattern. Basic examples of satellite knotting include the knot connected sum of knotted surfaces, and Artin's spinning construction [1925], as well as its twisted generalizations [Zeeman 1965; Litherland 1979]. Generally speaking, satellite knotting would lead to an increase of genus under certain natural assumptions such as nonzero winding number. However, this can be avoided if we are just concerned with knotted spheres or tori (see Section 4B). Like in the classical case, satellite knotting only changes the knot group by a van Kampen-type amalgamation. Therefore, it is usually an approach worth considering if one wishes to maintain some control on the group level during the construction. As far as we are concerned, the first explicit formulation of the satellite construction of $n$-knots in literature was due to Yaichi Shinohara [1971] in his paper about generalized Alexander polynomials and signatures; the satellite construction of knotted tori in $\mathbb{R}^{4}$ first appeared in Richard Litherland's paper [1981], where he studied the second homology of the knot group.

\section{Genera of slopes}

In this section, we introduce the genus and the singular genus for any slope of a knotted torus $K$ in $S^{4}$. We provide criteria about finiteness associated to the extendable subgroup $\mathscr{E}_{K}$ and the stable extendable subgroup $\mathscr{E}_{K}^{\mathrm{s}}$ of $\operatorname{Mod}\left(T^{2}\right)$ in terms of these notions.

3A. Genus and singular genus. Let $K: T^{2} \hookrightarrow S^{4}$ be a knotted torus in $S^{4}$, that is, a locally flat embedding of the torus into the 4-sphere. Let $X_{K}=S^{4}-K$ be the exterior of $K$ obtained by removing an open regular neighborhood of $K$.

Lemma 3.1. Let $F_{g}^{2}$ be the closed orientable surface of genus $g$, and $Y$ be a simply connected closed 4-manifold. Suppose $K: F_{g}^{2} \hookrightarrow Y$ is a null-homologous, locally flat embedding. Write $X=Y-K$ for the exterior of $K$ in $Y$. Then $\partial X$ is canonically homeomorphic to $F_{g}^{2} \times S^{1}$, up to isotopy, such that the homomorphism $H_{1}\left(F_{g}^{2}\right) \rightarrow H_{1}(X)$ induced by including $F_{g}^{2}$ as the first factor $F_{g}^{2} \times \mathrm{pt}$ is trivial. In 
particular, every essential simple closed curve $c \subset F_{g}^{2}$ bounds a locally flat, properly embedded, orientable compact surface $S \hookrightarrow X_{K}$ with $\partial S$ embedded as $c \times$ pt.

Proof. This is well-known, following from an easy homological argument. In fact, since $K$ is null-homologous, the normal bundle of $K$ in $Y$ is trivial, so $\partial X$ has a natural circle bundle structure $p: \partial X \rightarrow F_{g}^{2}$ over $F_{g}^{2}$, which splits. The splitting is given by framings of the normal bundle, which are in natural bijection with all the homomorphisms $\iota: H_{1}\left(F_{g}^{2}\right) \rightarrow H_{1}(\partial X)$ such that $p_{*} \circ \iota: H_{1}\left(F_{g}^{2}\right) \rightarrow H_{1}\left(F_{g}^{2}\right)$ is the identity. Using Poincaré duality and excision, it is easy to see $H^{1}(X) \cong \mathbb{Z}$ and $H^{1}(X, \partial X)=0$. Thus the homomorphism $H^{1}(X) \rightarrow H^{1}(\partial X)$ is injective, and the generator of $H_{1}(X)$ induces a homomorphism $\alpha: H_{1}(\partial X) \rightarrow \mathbb{Z}$. It is straightforward to check that $\alpha$ sends the circle-fiber of $\partial X$ to \pm 1 , so the kernel of $\alpha$ projects isomorphically onto $H_{1}\left(F_{g}^{2}\right)$ via $p_{*}$. This gives rise to the canonical splitting $\partial X=F_{g}^{2} \times S^{1}$. It follows clearly from the construction that $H_{1}\left(F_{g}^{2}\right) \rightarrow H_{1}(X)$ is trivial. Moreover, if $c \times \mathrm{pt}$ is an essential simple closed curve on $K \times \mathrm{pt}$, it is homologically trivial in $X$, so it represents an element $\left[a_{1}, b_{1}\right] \cdots\left[a_{k}, b_{k}\right]$ in the commutator subgroup of $\pi_{1}(X)$. We take a compact orientable surface $S^{\prime}$ of genus $k$ with exactly one boundary component, and there is a map $j: S^{\prime} \rightarrow X$ sending $\partial S^{\prime}$ homeomorphically onto $c \times$ pt. By a general position argument we may assume $j$ to be a locally flat proper immersion, and doing surgeries at double points yields a locally flat, properly embedded, orientable compact surface $S \hookrightarrow X$ bounded by $c \times$ pt.

This allows us to make the following definition:

Definition 3.2. Let $K: T^{2} \hookrightarrow S^{4}$ be a knotted torus. For any slope, that is, an essential simple closed curve, $c \subset K$, the genus $g_{K}(c)$ of $c$ is defined to be the minimum of the genus of $F$, as $F$ runs over all the locally flat, properly embedded, orientable, compact subsurfaces of $X_{K}$ bounded by $c \times \mathrm{pt} \subset \partial X_{K}$; see Lemma 3.1. The singular genus $g_{K}^{\star}(c)$ of $c$ is defined to be the minimum of the genus of $F$, as $F$ runs over all the compact orientable surfaces with connected nonempty boundary such that there is a continuous map $F \rightarrow X_{K}$ sending $\partial F$ homeomorphically onto $c \times$ pt.

Remark 3.3. Recall that for a group $G$ and any element $u$ in the commutator subgroup $[G, G]$, the commutator length $\mathrm{cl}(u)$ of $u$ is the smallest possible integer $k \geq 0$ such that $u$ can be written as a product of commutators $\left[a_{1}, b_{1}\right] \cdots\left[a_{k}, b_{k}\right]$, where $a_{i}, b_{i} \in G$, and $i=1, \ldots, k$. Note that elements of $[G, G]$ that are conjugate in $G$ have the same commutator length. As indicated in the proof of Lemma 3.1, it is clear that the singular genus $g_{K}^{\star}(c)$ is the commutator length $\mathrm{cl}(c)$, regarding $c$ as an element of the commutator subgroup of $\pi_{1}\left(X_{K}\right)$.

3B. Extendable subgroup and stable extendable subgroup. Let $\operatorname{Mod}\left(T^{2}\right)$ be the mapping class group of the torus, which consists of the isotopy classes of orientationpreserving self-homeomorphisms of $T^{2}$. Fixing a basis of $H_{1}\left(T^{2}\right)$, one can naturally 
identify $\operatorname{Mod}\left(T^{2}\right)$ as $\operatorname{SL}(2, \mathbb{Z})$. We often refer to the elements of $\operatorname{Mod}\left(T^{2}\right)$ as automorphisms of $T^{2}$, and do not distinguish elements of $\operatorname{Mod}\left(T^{2}\right)$ and their representatives.

For any knotted torus $K: T^{2} \hookrightarrow S^{4}$, an automorphism $\tau \in \operatorname{Mod}\left(T^{2}\right)$ is said to be extendable with respect to $K$ if $\tau$ can be extended as an orientation-preserving self-homeomorphism of $S^{4}$ via $K$. Note that this notion does not depend on the choice of the representative of $\tau$; see [Ding et al. 2012, Lemma 2.4]. It is also clear that all the extendable automorphisms form a subgroup of $\operatorname{Mod}\left(T^{2}\right)$.

Definition 3.4. For a knotted torus $K: T^{2} \hookrightarrow S^{4}$, the extendable subgroup with respect to $K$ is the subgroup of $\operatorname{Mod}\left(T^{2}\right)$ consisting of all the extendable automorphisms, denoted as $\mathscr{E}_{K} \leq \operatorname{Mod}\left(T^{2}\right)$.

The extendable subgroup $\mathscr{E}_{K}$ reflects some essential differences between knotted tori and knotted spheres (that is, 2-knots) in $S^{4}$. For instance, it is known that $\mathscr{E}_{K}$ is always a proper subgroup of $\operatorname{Mod}\left(T^{2}\right)$, of index at least three [Ding et al. 2012]; see [Montesinos 1983] for the diffeomorphism extension case. Moreover, index three is realized by any unknotted embedding, namely, one which bounds an embedded solid torus $S^{1} \times D^{2}$ in $S^{4}$ [Montesinos 1983]; see [Hirose 2002] for the general case of trivially embedded surfaces. In [Hirose 1993], $\mathscr{E}_{K}$ has been computed for the so-called spun $T^{2}$-knots and twisted spun $T^{2}$-knots. It is also clear that taking the connected sum with a knotted sphere in $S^{4}$ does not change the extendable subgroup. However, for a general knotted torus in $S^{4}$, the extendable subgroup $\mathscr{E}_{K}$ is poorly understood. In the following, we introduce a weaker notion called the stable extendable subgroup. From our point of view, the stable extendable subgroup is more closely related to the singular genera than the extendable subgroup is; see Section 6B.

Suppose $K: T^{2} \hookrightarrow S^{4}$ is a knotted torus in $S^{4}$, and $Y$ is a closed simply connected 4-manifold. There is a naturally induced embedding $K[Y]: T^{2} \hookrightarrow Y$ obtained by regarding $Y$ as the connected sum $S^{4} \# Y$ and embedding $T^{2}$ into the first summand via $K$. This is well defined up to isotopy, and we call $K[Y]$ the $Y$-stabilization of $K$. An automorphism $\tau \in \operatorname{Mod}\left(T^{2}\right)$ is said to be $Y$-stably extendable if $\tau$ extends over $Y$ as an orientation-preserving self-homeomorphism via $K[Y]$. All such automorphisms clearly form a subgroup of $\operatorname{Mod}\left(T^{2}\right)$. An automorphism $\tau \in \operatorname{Mod}\left(T^{2}\right)$ is said to be stably extendable if $\tau$ is $Y$-stably extendable for some closed simply connected 4-manifold $Y$. Note that if $\tau_{1}$ is $Y_{1}$-stably extendable and $\tau_{2}$ is $Y_{2}$-stably extendable, they are both $\left(Y_{1} \# Y_{2}\right)$-stably extendable. This means stably extendable automorphisms also form a subgroup of $\operatorname{Mod}\left(T^{2}\right)$.

Definition 3.5. For a knotted torus $K: T^{2} \hookrightarrow S^{4}$, the stable extendable subgroup with respect to $K$ is the subgroup of $\operatorname{Mod}\left(T^{2}\right)$ consisting of all the stably extendable automorphisms, denoted as $\mathscr{C}_{K}^{\mathscr{s}} \leq \operatorname{Mod}\left(T^{2}\right)$. 
Proposition 3.6. Let $K: T^{2} \hookrightarrow S^{4}$ be a knotted torus.

(1) If the singular genus $g_{K}^{\star}(c)$ takes infinitely many distinct values as $c$ runs over all the slopes of $K$, then the stable extendable subgroup $\mathscr{E}_{K}^{\mathrm{s}}$ is of infinite index in $\operatorname{Mod}\left(T^{2}\right)$.

(2) If there are at most finitely many distinct slopes $c \subset K$ with the singular genus $g_{K}^{\star}(c)$ at most $C$ for every $C>0$, then the stable extendable subgroup $\mathscr{E}_{K}^{\mathrm{s}}$ is finite.

Remark 3.7. Hence the same holds for the extendable subgroup $\mathscr{E}_{K}$. Using a similar argument, one can also show that the statements remain true when replacing $g_{K}^{\star}$ with $g_{K}$, and $\mathscr{E}_{K}^{\mathrm{s}}$ with $\mathscr{E}_{K}$.

Proof. First observe that the singular genus of a slope is invariant under the action of a stably extendable automorphism, namely, if $\tau \in \mathscr{C}_{K}^{\mathrm{s}}$, then $g_{K}^{\star}(c)=g_{K}^{\star}(\tau(c))$ for every slope $c \subset K$. This is clear because by the definition, $\tau$ extends over $X_{K}^{\prime}=X_{K} \# Y$ as a homeomorphism $\tilde{\tau}: X_{K}^{\prime} \rightarrow X_{K}^{\prime}$ for some simply connected closed 4-manifold $Y$. This induces an automorphism of $\pi_{1}\left(X_{K}^{\prime}\right) \cong \pi_{1}\left(X_{K}\right)$, which preserves the commutator length of $c$, or equivalently, the singular genus $g_{K}^{\star}(c)$ (Remark 3.3).

To see (1), note that $\operatorname{Mod}\left(T^{2}\right)$ acts transitively on the space $\mathscr{b}$ of all the slopes on $T^{2}$. It follows immediately from the invariance of singular genera above that the cardinality of value set of $g_{K}^{\star}$ is at most the index $\left[\operatorname{Mod}\left(T^{2}\right): \mathscr{E}_{K}^{\mathrm{s}}\right]$. Thus if the range of $g_{K}^{\star}$ is infinite, the index of $\mathscr{E}_{K}^{\mathrm{s}}$ in $\operatorname{Mod}\left(T^{2}\right)$ is also infinite.

To see (2), suppose $\tau \in \mathscr{C}_{K}^{\mathrm{s}}$. By the assumption and the invariance of the singular genus under $\tau$, for any slope $c \subset K$ there are at most finitely many distinct slopes in the sequence $c, \tau(c), \tau^{2}(c), \ldots$ Thus for some integers $k>l \geq 0, \tau^{k}(c)$ is isotopic to $\tau^{l}(c)$, or in other words, $\tau^{d}(c)$ is isotopic to $c$, where $d=k-l$. As $c$ is arbitrary, $\tau$ is a torsion element in $\operatorname{Mod}\left(T^{2}\right)$, so $\mathscr{E}_{K}^{\mathrm{s}}$ is a subgroup of $\operatorname{Mod}\left(T^{2}\right)$ consisting purely of torsion elements. It follows immediately that $\mathscr{E}_{K}^{\mathrm{s}}$ is a finite subgroup from the well-known fact that $\operatorname{Mod}\left(T^{2}\right) \cong \operatorname{SL}(2, \mathbb{Z})$ is virtually torsion-free. Indeed, the index of any finite-index torsion-free normal subgroup of $\operatorname{Mod}\left(T^{2}\right)$ yields an upper bound of the size of $\mathscr{C}_{K}^{\mathrm{s}}$.

\section{Induced seminorms on $H_{1}\left(T^{2} ; \mathbb{R}\right)$}

In this section, we introduce the seminorm $\|\cdot\|_{K}$ on $H_{1}\left(T^{2} ; \mathbb{R}\right)$ induced from any knotted torus $K: T^{2} \hookrightarrow S^{4}$. This may be regarded as a generalization of the (singular) Thurston norm in 3-dimensional topology. We prove a Schubert-type inequality in terms of seminorms associated with satellite constructions.

4A. The induced seminorm. There are various ways to formulate the induced seminorm, among which we shall take a more topological one. Suppose $K: T^{2} \hookrightarrow S^{4}$ 
is a knotted torus in $S^{4}$. We shall first define the value of $\|\cdot\|_{K}$ on $H_{1}\left(T^{2} ; \mathbb{Z}\right)$ then extend linearly and continuously over $H_{1}(K ; \mathbb{R})$.

Recall that for a connected orientable compact surface $F$, the complexity of $F$ is defined as $\chi_{-}(F)=\max \{-\chi(F), 0\}$. In general, for an orientable compact surface $F=F_{1} \sqcup \cdots \sqcup F_{s}$, the complexity of $F$ is defined as

$$
x(F)=\sum_{i=1}^{s} \chi_{-}\left(F_{i}\right) .
$$

For any $\gamma \in H_{1}\left(T^{2}\right)$, identified as an element of $H_{1}\left(\partial X_{K}\right)$, there exists a smooth immersion of pairs $(F, \partial F) \rightarrow\left(X_{K}, \partial X_{K}\right)$ such that $F$ is a (possibly disconnected) oriented compact surface, and that $\partial F$ represents $\gamma$. We define the complexity of $\gamma$ as

$$
x(\gamma)=\min _{F} x(F),
$$

where $F$ runs through all the possible immersed surfaces as described above.

The fact below follows immediately from the definition.

Lemma 4.1. With the notation above,

(1) $x(n \gamma) \leq n x(\gamma)$ for any $\gamma \in H_{1}\left(T^{2}\right)$ and any integer $n \geq 0$.

(2) $x\left(\gamma^{\prime}+\gamma^{\prime \prime}\right) \leq x\left(\gamma^{\prime}\right)+x\left(\gamma^{\prime \prime}\right)$ for any $\gamma^{\prime}, \gamma^{\prime \prime} \in H_{1}\left(T^{2}\right)$.

Definition 4.2. Let $K: T^{2} \hookrightarrow S^{2}$ be a knotted torus. For any $\gamma \in H_{1}\left(T^{2}\right)$, we define

$$
\|\gamma\|_{K}=\inf _{m \in \mathbb{Z}_{+}} \frac{x(m \gamma)}{m} .
$$

Lemma 4.3. (1) $\|n \gamma\|_{K}=n\|\gamma\|_{K}$ for any $\gamma \in H_{1}\left(T^{2}\right)$ and any integer $n \geq 0$.

(2) $\left\|\gamma^{\prime}+\gamma^{\prime \prime}\right\|_{K} \leq\left\|\gamma^{\prime}\right\|_{K}+\left\|\gamma^{\prime \prime}\right\|_{K}$ for any $\gamma^{\prime}, \gamma^{\prime \prime} \in H_{1}\left(T^{2}\right)$.

Proof. This follows from Lemma 4.1 and some elementary arguments. For any $\epsilon>0$, there is some $m>0$ such that $\|\gamma\|_{K}>(x(m \gamma) / m)-\epsilon$, and by Lemma 4.1,

$$
\frac{x(m \gamma)}{m}-\epsilon \geq \frac{x(n m \gamma)}{n m}-\epsilon \geq \frac{\|n \gamma\|_{K}}{n}-\epsilon .
$$

Letting $\epsilon \rightarrow 0$, we see $\|\gamma\|_{K} \geq\|n \gamma\|_{K} / n$. Moreover, for any $\epsilon>0$, there exists $m>0$ such that $\|n \gamma\|_{K}>(x(m n \gamma) / m)-\epsilon \geq n\|\gamma\|_{K}-\epsilon$. Letting $\epsilon \rightarrow 0$, we see $\|n \gamma\|_{K} \geq n\|\gamma\|_{K}$. This proves the first statement. To prove the second statement, for any $\epsilon>0$, there are $m^{\prime}, m^{\prime \prime}>0$ such that $\left\|\gamma^{\prime}\right\|_{K}>\left(x\left(m^{\prime} \gamma^{\prime}\right) / m^{\prime}\right)-\epsilon$ and $\left\|\gamma^{\prime \prime}\right\|_{K}>\left(x\left(m^{\prime \prime} \gamma^{\prime \prime}\right) / m^{\prime \prime}\right)-\epsilon$, so using Lemma 4.1,

$$
\begin{array}{r}
\left\|\gamma^{\prime}\right\|_{K}+\left\|\gamma^{\prime \prime}\right\|_{K}>\frac{x\left(m^{\prime} \gamma^{\prime}\right)}{m^{\prime}}+\frac{x\left(m^{\prime \prime} \gamma^{\prime \prime}\right)}{m^{\prime \prime}}-2 \epsilon \geq \frac{x\left(m^{\prime} m^{\prime \prime} \gamma^{\prime}\right)}{m^{\prime} m^{\prime \prime}}+\frac{x\left(m^{\prime} m^{\prime \prime} \gamma^{\prime \prime}\right)}{m^{\prime} m^{\prime \prime}}-2 \epsilon \\
\geq \frac{x\left(m^{\prime} m^{\prime \prime}\left(\gamma^{\prime}+\gamma^{\prime \prime}\right)\right)}{m^{\prime} m^{\prime \prime}}-2 \epsilon \geq\left\|\gamma^{\prime}+\gamma^{\prime \prime}\right\|_{K}-2 \epsilon .
\end{array}
$$


Letting $\epsilon \rightarrow 0$, we see the second statement.

By Lemma 4.3 , we can extend $\|\cdot\|_{K}$ radially over $H_{1}\left(T^{2} ; \mathbb{Q}\right)$, then extend continuously over $H_{1}\left(T^{2} ; \mathbb{R}\right)$. This uniquely defines a seminorm

$$
\|\cdot\|_{K}: H_{1}\left(T^{2} ; \mathbb{R}\right) \rightarrow[0,+\infty) .
$$

Recall a seminorm on a real vector space $V$ is a function $\|\cdot\|: V \rightarrow[0,+\infty)$ such that $\|r v\|=|r|\|v\|$ for any $r \in \mathbb{R}, v \in V$, and that $\left\|v^{\prime}+v^{\prime \prime}\right\| \leq\left\|v^{\prime}\right\|+\left\|v^{\prime \prime}\right\|$ for any $v^{\prime}, v^{\prime \prime} \in V$. It is a norm if it is in addition positive-definite, namely $\|v\|=0$ if and only if $v \in V$ is zero.

Definition 4.4. Let $K: T^{2} \hookrightarrow S^{4}$ be a knotted torus, and $c \subset T^{2}$ be a slope. Then the seminorm $\|c\|_{K}$ is defined as $\|[c]\|_{K}$, where $[c] \in H_{1}\left(T^{2}\right)$.

Remark 4.5. Recall that for a group $G$ and any element $u$ in the commutator subgroup $[G, G]$, the stable commutator length is

$$
\operatorname{scl}(u)=\lim _{n \rightarrow+\infty} \frac{\operatorname{cl}\left(u^{n}\right)}{n},
$$

where $\operatorname{cl}(\cdot)$ denotes the commutator length (Remark 3.3). It is not hard to see that for any slope $c \subset K$, the seminorm $\|c\|_{K}$ equals $\operatorname{scl}(c)$, regarding $c$ as an element of the commutator subgroup of $\pi_{1}\left(X_{K}\right)$; see [Calegari 2009, Proposition 2.10].

The lemma below follows immediately from the definition and Proposition 3.6:

Lemma 4.6. If $c \subset K$ is a slope with $\|c\|_{K}>0$, then $g_{K}^{\star}(c) \geq\left(\|c\|_{K}+1\right) / 2$. Hence the stable extendable subgroup $\mathscr{E}_{K}^{\mathrm{s}}$ is finite if $\|\cdot\|_{K}$ is nondegenerate. The same holds if we replace $g_{K}^{\star}$ with $g_{K}$ and $\mathscr{E}_{K}^{\mathrm{s}}$ with $\mathscr{E}_{K}$.

4B. The satellite construction. The satellite construction for knotted tori is analogous to that of classical knots in $S^{3}$; see Section $2 \mathrm{C}$ for historical remarks.

Fix a product structure of $T^{2} \cong S^{1} \times S^{1}$. We shall denote the thickened torus with the standard parametrization as

$$
\Theta^{4}=S^{1} \times S^{1} \times D^{2}
$$

The standard unknotted torus $T_{\text {std }}: T^{2} \subset S^{4}$ is a smoothly embedded torus such that $T_{\text {std }}$ bounds two smoothly embedded solid tori $D^{2} \times S^{1}$ and $S^{1} \times D^{2}$ in $S^{4}$, respective to factors. It is unique up to diffeotopy of $S^{4}$. Let $K_{\mathrm{c}}: T^{2} \hookrightarrow S^{4}$ be a knotted torus. There is a natural trivial product structure on a compact tubular neighborhood $\mathcal{N}\left(K_{\mathrm{c}}\right) \cong T^{2} \times D^{2}$ of $K_{\mathrm{c}}$, so that $c \times *$ is homologically trivial in the complement $X_{K_{\mathrm{c}}}$ for any slope $c \subset T^{2}$. Thus there is a natural isomorphism $\mathcal{N}\left(K_{\mathrm{c}}\right) \cong \Theta^{4}$, up to isotopy, as we fixed the product structure on $T^{2}$. 
Definition 4.7. A pattern knotted torus is a smooth embedding $K_{\mathrm{p}}: T^{2} \hookrightarrow \Theta^{4}$. The winding number $w\left(K_{\mathrm{p}}\right)$ of $K_{\mathrm{p}}$ is the algebraic intersection number of $\left[K_{\mathrm{p}}\right] \in \mathrm{H}_{2}\left(\Theta^{4}\right)$ and the fiber disk $\left[\mathrm{pt} \times \mathrm{pt} \times D^{2}\right] \in H_{2}\left(\Theta^{4}, \partial \Theta^{4}\right)$.

Definition 4.8. Let $K_{\mathrm{c}}: T^{2} \hookrightarrow S^{4}$ be a knotted torus and $K_{\mathrm{p}}: T^{2} \hookrightarrow \Theta^{4}$ be a pattern knotted torus. After fixing a product structure on $T^{2}$, the satellite knotted torus, denoted as $K=K_{\mathrm{c}} \cdot K_{\mathrm{p}}$, is the composition

$$
T^{2} \stackrel{K_{\mathrm{p}}}{\longrightarrow} \Theta^{4} \stackrel{\cong}{\longrightarrow} \mathcal{N}\left(K_{\mathrm{c}}\right) \stackrel{\subset}{\longrightarrow} S^{4} .
$$

We call $K_{\mathrm{c}}$ the companion knotted torus. The desatellite $\hat{K}_{\mathrm{p}}: T^{2} \hookrightarrow S^{4}$ of $K$ is the knotted torus $\hat{K}_{\mathrm{p}}=T_{\text {std }} \cdot K_{\mathrm{p}}$.

For any element $\gamma \in H_{1}\left(T^{2}\right)$ and a pattern $K_{\mathrm{p}}: T^{2} \hookrightarrow \Theta^{4}$, there is a push-forward element $\gamma_{\mathrm{c}} \in H_{1}\left(T^{2}\right)$ under the composition:

$$
T^{2} \stackrel{K_{\mathrm{p}}}{\longrightarrow} \Theta^{4} \stackrel{\cong}{\longrightarrow} T^{2} \times D^{2} \rightarrow T^{2},
$$

where the isomorphism respects the choice of the product structure on $T^{2}$, and the last map is the projection onto the $T^{2}$ factor. If $K=K_{\mathrm{c}} \cdot K_{\mathrm{p}}$ is a satellite with pattern $K_{\mathrm{p}}$, one should regard $\gamma$ as an element of $H_{1}(K)$, and $\gamma_{\mathrm{c}}$ as an element of $H_{1}\left(K_{\mathrm{c}}\right)$.

4C. A Schubert-type inequality. The theorem below is analogous to the Schubert inequality in classical knot theory [Schubert 1953, Kapitel II, §12].

Theorem 4.9. Suppose $K=K_{\mathrm{c}} \cdot K_{\mathrm{p}}$ is a satellite knotted torus in $S^{4}$. Then for any $\gamma \in H_{1}\left(T^{2} ; \mathbb{R}\right),\|\gamma\|_{K} \geq\|\gamma\|_{\hat{K}_{\mathrm{p}}}$. Moreover, if the winding number $w\left(K_{\mathrm{p}}\right)$ is nonzero, then $\|\gamma\|_{K} \geq\|\gamma\|_{\hat{K}_{\mathrm{p}}}+\left\|\gamma_{\mathrm{c}}\right\|_{K_{\mathrm{c}}}$.

We prove Theorem 4.9 in the rest of this subsection.

Let $X_{K}$ be the complement of the satellite knot $K=K_{\mathrm{c}} \cdot K_{\mathrm{p}}$ in $S^{4}$. The satellite construction gives a decomposition $X_{K}=Y \cup X_{K_{\mathrm{c}}}$, glued along the image of $\partial \Theta^{4}$. $Y$ is diffeomorphic to the complement of $K_{\mathrm{p}}$ in $\Theta^{4}$, so it has two boundary components, namely the satellite boundary $\partial_{\mathrm{S}} Y$, which is $\partial X_{K}$, and the companion boundary $\partial_{\mathrm{c}} Y$ which is the image of $\partial \Theta^{4}$.

Similarly, the complement $X_{\hat{K}_{\mathrm{p}}}$ can be decomposed as $Y \cup X_{T_{\text {std }}}$.

The first inequality is proved in the following lemma:

Lemma 4.10. $\|\gamma\|_{K} \geq\|\gamma\|_{\hat{K}_{\mathrm{p}}}$.

Proof. We equip $X_{K_{\mathrm{c}}}$ with a finite $\mathrm{CW}$ complex structure such that there is only one 0 -cell and the 0 -cell is contained in $\partial X_{K_{\mathrm{c}}}$, which is a subcomplex of $X_{K_{\mathrm{c}}}$. Let $X_{K_{\mathrm{c}}}^{(q)}$ be the union of $\partial X_{K_{\mathrm{c}}}$ and the $q$-skeleton of $X_{K_{\mathrm{c}}}$. We may extend the identity map on $Y$ to a continuous map $f: Y \cup X_{K_{\mathrm{c}}}^{(2)} \rightarrow X_{\hat{K}_{\mathrm{p}}}$. To see this, note that the inclusion map 
$\partial X_{K} \rightarrow X_{K}$ induces a surjective map on $H_{1}$ for any $K: T^{2} \rightarrow S^{4}$, so the identity map on $\partial X_{K_{\mathrm{c}}}$ induces a natural isomorphism $H_{1}\left(X_{K_{\mathrm{c}}}\right) \cong H_{1}\left(X_{T_{\mathrm{std}}}\right)$. Since every 1-cell in $X_{K_{\mathrm{c}}}$ represents a 1-cycle, we can extend $\operatorname{id}_{\partial_{\mathrm{c}} Y}$ to a map $f \mid: X_{K_{\mathrm{c}}}^{(1)} \rightarrow X_{T_{\mathrm{std}}}$, so that the induced map $H_{1}\left(X_{K_{\mathrm{c}}}^{(1)}\right) \rightarrow H_{1}\left(X_{T_{\mathrm{std}}}\right)$ agrees with the map on the first homology induced by $X_{K_{\mathrm{c}}}^{(1)} \hookrightarrow X_{K_{\mathrm{c}}}$. It is easy to see $X_{T_{\mathrm{std}}} \simeq S^{1} \vee S^{2} \vee S^{2}$, so $\pi_{1}\left(X_{T_{\text {std }}}\right) \cong \mathbb{Z}$. Hence the previous $f \mid$ can be further extended as $f \mid: X_{K_{\mathrm{c}}}^{(2)} \rightarrow X_{T_{\text {std }}}$ since the boundary of any 2-cell is mapped to a null-homotopic loop in $X_{T_{\text {std }}}$ by the construction.

Thus we obtain a map $f: Y \cup X_{K_{\mathrm{c}}}^{(2)} \rightarrow X_{\hat{K}_{\mathrm{p}}}$ by the map above and the identity on $Y$. Let $j: F \rightarrow X_{K}$ be an immersed compact orientable surface such that $j(\partial F) \subset \partial X_{K}$. We may assume $F$ meets $\partial_{\mathrm{c}} Y$ transversely. We homotope $j$ to $j^{\prime}: F \rightarrow Y \cup X_{K_{\mathrm{c}}}^{(2)}$. Then we obtain a map $f \circ j^{\prime}: F \rightarrow X_{\hat{K}_{\mathrm{p}}}$ which may be homotoped to an immersion. As $F$ is arbitrary, this implies $\|\gamma\|_{K} \geq\|\gamma\|_{\hat{K}_{\mathrm{p}}}$ by the definition of the seminorm.

Now we consider the case when $w\left(K_{\mathrm{p}}\right) \neq 0$. The image of $\mathrm{pt} \times \mathrm{pt} \times \partial D^{2} \subset Y$ under the natural inclusion $Y \subset X_{K}$ will be denoted $\mu_{\mathrm{c}}$. We call $\mu_{\mathrm{c}}$ the companion meridian. The following lemma follows immediately from the construction:

Lemma 4.11. Identify $H_{1}\left(X_{K_{\mathrm{c}}}\right) \cong \mathbb{Z}$ and $H_{1}\left(X_{K}\right) \cong \mathbb{Z}$. Then $H_{1}\left(X_{K_{\mathrm{c}}}\right) \rightarrow H_{1}\left(X_{K}\right)$ is multiplication by $w\left(K_{\mathrm{p}}\right)$.

Proof. Note $\mu_{\mathrm{c}}$ represents a generator of $H_{1}\left(X_{K_{\mathrm{c}}}\right)$. By definition of $w\left(K_{\mathrm{p}}\right), \mu_{\mathrm{c}}$ is homologous to $w\left(K_{\mathrm{p}}\right)$ times the meridian of $K$. The lemma follows as the meridian of $K$ generates $H_{1}\left(X_{K}\right) \cong \mathbb{Z}$ by Alexander duality.

Lemma 4.12. If $w\left(K_{\mathrm{p}}\right) \neq 0$, then the inclusion map $\partial_{\mathrm{c}} Y \subset Y$ induces an injective homomorphism $H_{1}\left(\partial_{\mathrm{c}} Y\right) \rightarrow H_{1}(Y)$. In particular, the inclusion map $\partial_{\mathrm{c}} Y \subset Y$ is $\pi_{1}$-injective.

Proof. By the long exact sequence

$$
\cdots \rightarrow H_{2}\left(Y, \partial_{\mathrm{c}} Y\right) \rightarrow H_{1}\left(\partial_{\mathrm{c}} Y\right) \rightarrow H_{1}(Y) \rightarrow \cdots,
$$

it suffices to show $H_{2}\left(Y, \partial_{\mathrm{c}} Y\right)$ is finite, since $H_{1}\left(\partial_{\mathrm{c}} Y\right) \cong H_{1}\left(\partial \Theta^{4}\right)$ is torsion-free. By the Poincaré-Lefschetz duality and excision,

$$
H_{2}\left(Y, \partial_{\mathrm{c}} Y\right) \cong H^{2}\left(Y, \partial_{\mathrm{s}} Y\right) \cong H^{2}\left(\Theta^{4}, K_{\mathrm{p}}\right) .
$$

The long exact sequence

$$
\cdots \rightarrow H^{1}\left(\Theta^{4}\right) \rightarrow H^{1}\left(K_{\mathrm{p}}\right) \rightarrow H^{2}\left(\Theta^{4}, K_{\mathrm{p}}\right) \rightarrow H^{2}\left(\Theta^{4}\right) \rightarrow H^{2}\left(K_{p}\right) \rightarrow \cdots
$$

is induced by the inclusion $K_{\mathrm{p}} \subset \Theta^{4}$, (or equivalently by $K_{\mathrm{p}}: T^{2} \hookrightarrow \Theta^{4}$ ). Since $\Theta^{4} \simeq T^{2}, K_{\mathrm{p}}$ induces a map $h: T^{2} \rightarrow T^{2}$. It is also clear that $w\left(K_{\mathrm{p}}\right)$ is the degree of $h$. Since $w\left(K_{\mathrm{p}}\right) \neq 0$, it is clear that the map $h^{*}: H^{*}\left(T^{2}\right) \rightarrow H^{*}\left(T^{2}\right)$ is injective 
on all dimensions, so must be $H^{*}\left(\Theta^{4}\right) \rightarrow H^{*}\left(K_{\mathrm{p}}\right)$. Thus $H^{2}\left(\Theta^{4}, K_{\mathrm{p}}\right)$ is finite from the long exact sequence. We conclude $H_{2}\left(Y, \partial_{\mathrm{c}} Y\right)$ is finite as desired.

Note it suffices to prove Theorem 4.9 for $\gamma \in H_{1}\left(T^{2} ; \mathbb{Z}\right)$. Remember that we regard $\gamma$ as in $H_{1}(K)$, identified as the kernel of $H_{1}\left(\partial X_{K}\right) \rightarrow H_{1}\left(X_{K}\right)$. For any $\epsilon>0$, let $j: F \leftrightarrow X_{K}$ be a properly immersed orientable compact (possibly disconnected) surface, that is, $j^{-1}\left(\partial X_{K}\right)=\partial F$, such that $j_{*}[\partial F]=m \gamma$ for some integer $m>0$, and that

$$
\|\gamma\|_{K} \leq \frac{x(F)}{m}<\|\gamma\|_{K}+\epsilon .
$$

We may assume $F$ has no disk or closed component, so $x(F)=-\chi(F)$. We may also assume $F$ intersects $\partial_{\mathrm{c}} Y$ transversely, so $j^{-1}\left(\partial_{\mathrm{c}} Y\right)$ is a disjoint union of simple closed curves on $F$. Write $F_{\mathrm{p}}, F_{\mathrm{c}}$ for $j^{-1}(Y), j^{-1}\left(X_{K_{\mathrm{c}}}\right)$, respectively.

Lemma 4.13. Suppose $w\left(K_{\mathrm{p}}\right) \neq 0$. If $V$ is a component of $F_{\mathrm{p}}$ where $j(\partial V) \subset \partial_{\mathrm{c}} Y$, then there is a map $j^{\prime} \mid: V \rightarrow \partial_{\mathrm{c}} Y$, such that $\left.j^{\prime}\right|_{\partial V}=j$.

Proof. We may take a collection of embedded arcs $u_{1}, \ldots, u_{n}$ whose endpoints lie on $\partial V$, cutting $V$ into a disk $D$. This gives a cellular decomposition of $V$. We may first extend the map $\left.j\right|_{\partial V}: \partial V \rightarrow \partial_{\mathrm{c}} Y$ to a map $\left.j^{\prime}\right|_{V^{(1)}}$ over the 1-skeleton of $V$. Let $\phi: \partial D \rightarrow V^{(1)}$ be the attaching map. We have $j_{*}^{\prime} \phi_{*}[\partial D]=j_{*}[\partial V]$ in $H_{1}\left(\partial_{\mathrm{c}} Y\right)$ by the construction. As $w\left(K_{\mathrm{p}}\right) \neq 0$, by Lemma 4.12, $H_{1}\left(\partial_{\mathrm{c}} Y\right) \rightarrow H_{1}(Y)$ is an injective homomorphism, so $j_{*}[\partial V]=0$ in $H_{1}\left(\partial_{\mathrm{c}} Y\right)$ since it is bounded by $j_{*}[V]$. Thus $j_{*}^{\prime} \phi_{*}[\partial D]=0$ in $H_{1}\left(\partial_{\mathrm{c}} Y\right)$, and hence $\partial D$ is null-homotopic in $\partial_{\mathrm{c}} Y$ under $j^{\prime} \circ \phi$ as $\pi_{1}\left(\partial_{\mathrm{c}} Y\right) \cong H_{1}\left(\partial_{\mathrm{c}} Y\right)$, (remember $\partial_{\mathrm{c}} Y \cong \partial \Theta^{4}$ is a 3-torus). Therefore, we may extend $\left.j^{\prime}\right|_{V^{(1)}}$ further over $D$ to obtain $j^{\prime}: V \rightarrow \partial_{\mathrm{c}} Y$ as desired.

Lemma 4.14. We may modify $j: F \rightarrow X_{K}$ within the interior of $F$ so that every component of $j^{-1}\left(\partial_{\mathrm{c}} Y\right)$ that is inessential on $F$ bounds a disk component of $j^{-1}\left(X_{K_{\mathrm{c}}}\right)$.

Proof. Let $a \subset j^{-1}\left(\partial_{\mathrm{c}} Y\right)$ be a component inessential on $F$, and $D \subset F$ be an embedded disk whose boundary is $a$. If $D$ is not contained in $F_{\mathrm{c}}$, then $D \cap F_{\mathrm{p}} \neq \varnothing$. Any component of $D \cap F_{\mathrm{p}}$ must have all its boundary components lying on $j^{-1}\left(\partial_{\mathrm{c}} Y\right)$. By Lemma 4.13, we may redefine $j$ on these components relative to boundary so that they are all mapped into $X_{\mathrm{c}}$. After this modification and a small perturbation, either $a$ disappears from $j^{-1}\left(\partial_{\mathrm{c}} Y\right)$ (if $\left.\partial D \subset D \cap F_{\mathrm{p}}\right)$, or at least one component of $j^{-1}\left(\partial_{\mathrm{c}} Y\right)$ in the interior of $D$ disappears (if $\partial D \subset D \cap F_{\mathrm{c}}$ ). Thus the number of inessential components of $j^{-1}\left(\partial_{\mathrm{c}} Y\right)$ decreases strictly under this modification. Therefore, after at most finitely many such modifications, every inessential component of $j^{-1}\left(\partial_{\mathrm{c}} Y\right)$ bounds a disk component of $F_{\mathrm{c}}$.

Without loss of generality, we assume that $j: F \leftrightarrow X_{K}$ satisfies the conclusion of Lemma 4.14. 
Lemma 4.15. There is a finite cyclic covering $\kappa: \tilde{F} \rightarrow F$ such that for every essential component $a \in j^{-1}\left(\partial_{\mathrm{c}} Y\right)$ with $[j(a)] \neq 0$ in $H_{1}\left(X_{K}\right)$, and every component $\tilde{a}$ of $\kappa^{-1}(a)$, the image $j(\kappa(\tilde{a}))$ represents the same element in $H_{1}\left(X_{K}\right) \cong \mathbb{Z}$ up to sign.

Proof. Let $a_{1}, \ldots, a_{s}$ be all the essential components $j^{-1}\left(\partial_{\mathrm{c}} Y\right)$ such that $\left[j\left(a_{i}\right)\right] \neq 0$ in $H_{1}\left(X_{K}\right) \cong \mathbb{Z}$. Let $d>0$ be the least common multiple of all the $\left[j\left(a_{i}\right)\right]$. Consider the covering $\kappa: \tilde{F} \rightarrow F$ corresponding to the preimage of the subgroup $d \cdot H_{1}\left(X_{K}\right)$ under $\pi_{1}(F) \rightarrow \pi_{1}\left(X_{K}\right) \rightarrow H_{1}\left(X_{K}\right)$. It is straightforward to check that $\kappa$ satisfies the conclusion.

Let $\kappa: \tilde{F} \rightarrow F$ be a covering as obtained in Lemma 4.15. Let $d>0$ be the degree of $\kappa$, so $x(\tilde{F})=d x(F)$. Clearly $j_{*} \kappa_{*}[\partial \tilde{F}]=m d \gamma$, and also

$$
\|\gamma\|_{K} \leq \frac{x(\tilde{F})}{m d}<\|\gamma\|_{K}+\epsilon
$$

Moreover, as any inessential component of $j^{-1}\left(\partial_{\mathrm{c}} Y\right)$ bounds a disk component of $F_{\mathrm{c}}$, it is clear that any inessential component of $(j \circ \kappa)^{-1}\left(\partial_{\mathrm{c}} Y\right)$ bounds a disk component of $\tilde{F}_{\mathrm{c}}=\kappa^{-1}\left(F_{\mathrm{c}}\right)$.

Therefore, instead of using $j: F \rightarrow X_{K}$, we may use $j \circ \kappa: \tilde{F} \leftrightarrow X_{K}$ as well. From now on, we rewrite $j \circ \kappa$ as $j, \tilde{F}$ as $F$, and $m d$ as $m$, so $j: F \leftrightarrow X_{K}$ satisfies the conclusions of Lemmas 4.14, 4.15.

Let $Q \subset F_{\mathrm{c}}$ be the union of the disk components of $F_{\mathrm{c}}$. Let $F_{\mathrm{c}}^{\prime}$ be $F_{\mathrm{c}}-Q$, and $F_{\mathrm{p}}^{\prime}$ be $F_{\mathrm{p}} \cup Q$ (glued up along adjacent boundary components). We have the decompositions

$$
F=F_{\mathrm{p}} \cup F_{\mathrm{c}}=F_{\mathrm{p}}^{\prime} \cup F_{\mathrm{c}}^{\prime} .
$$

Moreover, there is no inessential component of $\partial F_{\mathrm{c}}^{\prime}$ by our assumption on $F$, so $F_{\mathrm{c}}^{\prime}$ and $F_{\mathrm{p}}^{\prime}$ are essential subsurfaces of $F$ (that is, whose boundary components are essential).

Lemma 4.16. Suppose $F$ is a compact orientable surface with no disk or sphere component, and $E_{1}, E_{2}$ are essential compact subsurfaces of $F$ with disjoint interiors such that $F=E_{1} \cup E_{2}$. Then $x(F)=x\left(E_{1}\right)+x\left(E_{2}\right)$.

Proof. Note $\chi(F)=\chi\left(E_{1}\right)+\chi\left(E_{2}\right)$. As each $E_{i}$ is essential, there is no disk component of $E_{i}$, and by the assumption there is no sphere component, either. Thus, for each component $C$ of $E_{i}, x(C)=-\chi(C)$. We have $x(F)=x\left(E_{1}\right)+x\left(E_{2}\right)$.

The desatellite term in Theorem 4.9 comes from the following construction.

Lemma 4.17. Under the assumptions above, there is a properly immersed compact orientable surface $\hat{j}: \hat{F}_{\mathrm{p}}^{\prime} \rightarrow X_{\hat{K}_{\mathrm{p}}}$ such that $x\left(\hat{F}_{\mathrm{p}}^{\prime}\right) \leq x\left(F_{\mathrm{p}}^{\prime}\right)$, and that $\hat{j}_{*}\left[\partial \hat{F}_{\mathrm{p}}^{\prime}\right]=m \gamma$ in $H_{1}\left(T^{2}\right)$. 
Proof. As $F$ has been assumed to satisfy the conclusion of Lemma 4.15, there is an $\omega \in H_{1}\left(X_{K}\right)$ such that every component of $\partial_{\mathrm{c}} F_{\mathrm{p}}^{\prime}$ (that is, $F_{\mathrm{p}}^{\prime} \cap j^{-1}\left(\partial_{\mathrm{c}} Y\right)$ ) represents either $\pm \omega$ or 0 , and the algebraic sum over all the components is zero since they bound $j\left(F_{\mathrm{c}}^{\prime}\right) \subset X_{K}$. Thus we may assume there are $s$ components representing 0 , $t$ components representing $\omega$, and $t$ components representing $-\omega$, where $s, t \geq 0$. We construct $\hat{F}_{\mathrm{p}}^{\prime}$ by attaching $s$ disks and $t$ annuli to $\partial_{\mathrm{c}} F_{\mathrm{p}}^{\prime}$, such that each disk is attached to a component representing 0 , and each annulus is attached to a pair of components representing opposite $\pm \omega$-classes. Let $\mathscr{D}$ be the union of attached disks, and $\mathscr{A}$ be the union of attached annuli. The result is a compact orientable surface $\hat{F}_{\mathrm{p}}^{\prime}=F_{\mathrm{p}}^{\prime} \cup \mathscr{D} \cup \mathscr{A}$ such that $\partial \hat{F}_{\mathrm{p}}^{\prime} \cong \partial F$. It is clear that $x\left(\hat{F}_{\mathrm{p}}^{\prime}\right) \leq x\left(F_{\mathrm{p}}^{\prime} \cup \mathscr{A}\right)=x\left(F_{\mathrm{p}}^{\prime}\right)$, (see Lemma 4.16).

To construct $\hat{j}$, we extend the map

$$
j \mid: F_{\mathrm{p}} \rightarrow Y \subset X_{\hat{K}_{\mathrm{p}}}=Y \cup X_{T_{\mathrm{std}}}
$$

over $\hat{F}_{\mathrm{p}}=F_{\mathrm{p}} \cup Q \cup \mathscr{D} \cup \mathscr{A}$, using the fact that $\pi_{1}\left(X_{T_{\mathrm{std}}}\right) \cong H_{1}\left(X_{T_{\text {std }}}\right) \cong \mathbb{Z}$. Specifically, to extend the map over $Q$, let $s$ be a component of $\partial_{\mathrm{c}} F_{\mathrm{p}}$ bounding a disk component of $Q$. Then $j_{*}[s]=0$ in $H_{1}\left(X_{K}\right)$. Hence it lies in the subgroup $H_{1}\left(T^{2} \times \mathrm{pt}\right)$ of $H_{1}\left(\partial \Theta^{4}\right) \cong H_{1}\left(\partial_{\mathrm{c}} Y\right)$, and by the desatellite construction, $\hat{j}(s)$ should also be null-homologous in $X_{T_{\text {std }}}$. We can extend $\hat{j}$ over the disk $D \subset Q$ bounded by $s$. After extending for every component of $Q$, we obtain

$$
\hat{j} \mid: F_{\mathrm{p}} \cup Q \rightarrow X_{\hat{K}_{\mathrm{p}}}
$$

Similarly, we may extend $\hat{j} \mid$ over $\mathscr{D}$. To extend over $\mathscr{A}$, let $A \subset \mathscr{A}$ be an attached annulus component as in the construction. Let $\partial A=s_{+} \sqcup s_{-}$such that $j_{*}\left[s_{ \pm}\right]= \pm \omega$ in $H_{1}\left(X_{K}\right)$. By the desatellite construction, $\hat{j}_{*}\left[s_{ \pm}\right]= \pm \omega$ in $H_{1}\left(X_{T_{\text {std }}}\right)$. Since $\pi_{1}\left(X_{T_{\mathrm{std}}}\right) \cong H_{1}\left(X_{T_{\mathrm{std}}}\right), \hat{j}\left(s_{+}\right)$is free-homotopic to the orientation-reversal of $\hat{j}\left(s_{-}\right)$. In other words, we can extend $\hat{j} \mid$ over $A$. After extending for every attached annulus, we obtain $\hat{j}: \hat{F}_{\mathrm{p}}^{\prime} \rightarrow X_{\hat{K}_{\mathrm{p}}}$.

Since $\left.\hat{j}\right|_{\partial \hat{F}_{\mathrm{p}}}$ is the same as $\left.j\right|_{\partial F}$ under the natural identification $\hat{j}_{*}\left[\partial \hat{F}_{\mathrm{p}}^{\prime}\right]=m \gamma$ in $H_{1}\left(T^{2}\right)$ (where $H_{1}\left(T^{2}\right)$ may be regarded as either $H_{1}(K)$ or $H_{1}\left(\hat{K}_{\mathrm{p}}\right)$ under the natural identification), after homotoping $\hat{j}: \hat{F}_{\mathrm{p}}^{\prime} \rightarrow X_{\hat{K}_{\mathrm{p}}}$ to a smooth immersion, we obtain the map as desired.

The contribution of the companion term in Theorem 4.9 basically comes from $F_{\mathrm{c}}^{\prime}$. However, $j_{*}\left[F_{\mathrm{c}}^{\prime}\right]$ does not necessarily represent $m \gamma_{\mathrm{c}}$, but may differ by a term of zero $\|\cdot\|_{K_{\mathrm{c}}}$-seminorm.

To be precise, note the image of any component of $\partial Q \subset \partial_{\mathrm{c}} Y$ under $j$ lies in the kernel of $H_{1}\left(\partial_{\mathrm{c}} Y\right) \rightarrow H_{1}\left(X_{K_{\mathrm{c}}}\right)$, which we may identify with $H_{1}\left(K_{\mathrm{c}}\right)$. Thus $\alpha=j_{*}[\partial Q] \in H_{1}\left(\partial_{\mathrm{c}} Y\right)$ lies in $H_{1}\left(K_{\mathrm{c}}\right)$. Also, $j_{*}\left[\partial F_{\mathrm{c}}\right]=m \gamma_{\mathrm{c}} \in H_{1}\left(K_{\mathrm{c}}\right)<H_{1}\left(\partial_{\mathrm{c}} Y\right)$. 
Thus $\beta=m \gamma_{\mathrm{c}}-\alpha$ in $H_{1}\left(K_{\mathrm{c}}\right)<H_{1}\left(\partial_{\mathrm{c}} Y\right)$ is represented by $j_{*}\left[F_{\mathrm{c}}^{\prime}\right]$. We have

$$
m \gamma_{\mathrm{c}}=\alpha+\beta \text {. }
$$

Lemma 4.18. With the notation above, $\|\alpha\|_{K_{\mathrm{c}}}=0$, and hence $m\left\|\gamma_{\mathrm{c}}\right\|_{K_{\mathrm{c}}}=\|\beta\|_{K_{\mathrm{c}}}$.

Proof. For any component $s \subset \partial Q, s$ bounds an embedded disk component $D$ of $Q \subset F_{\mathrm{c}}$ by the definition of $Q$. It follows that $j(s)$ is null-homotopic in $X_{K_{\mathrm{c}}}$, and hence $\left\|j_{*}[s]\right\|_{K_{\mathrm{c}}}=0$. As this works for any component of $\partial Q$, we see $\|\alpha\|_{K_{\mathrm{c}}}=\left\|j_{*}[\partial Q]\right\|_{K_{\mathrm{c}}}=0$. The "hence" part follows from that $\|\cdot\|_{K_{\mathrm{c}}}$ is a seminorm on $H_{1}\left(K_{\mathrm{c}} ; \mathbb{R}\right)$.

Proof of Theorem 4.9. The first inequality follows from Lemma 4.10. In the rest, we assume $w\left(K_{\mathrm{p}}\right) \neq 0$. Let $j: F \rightarrow X_{K}$ be a surface that $\epsilon$-approximates $\|\gamma\|_{K}$ as before. We may assume $j$ satisfies the conclusion of Lemma 4.14 possibly after a modification. Possibly after passing to a finite cyclic covering of $F$, we may further assume $j$ satisfies the conclusion of Lemma 4.15 as we have explained. We have the decomposition $F=F_{\mathrm{p}}^{\prime} \cup F_{\mathrm{c}}^{\prime}$ of $F$ into essential subsurfaces, so by Lemma 4.16, $x(F)=x\left(F_{\mathrm{p}}^{\prime}\right)+x\left(F_{\mathrm{c}}^{\prime}\right)$. By Lemma 4.17, there is an immersed surface $\hat{j}: \hat{F}_{\mathrm{p}}^{\prime} \rightarrow X_{\hat{K}_{\mathrm{p}}}$ representing $m \gamma$ in $H_{1}\left(\hat{K}_{\mathrm{p}}\right)$, with $x\left(\hat{F}_{\mathrm{p}}^{\prime}\right) \leq x\left(F_{\mathrm{p}}^{\prime}\right)$, so

$$
x\left(F_{\mathrm{p}}^{\prime}\right) \geq x\left(\hat{F}_{\mathrm{p}}^{\prime}\right) \geq m\|\gamma\|_{\hat{K}_{\mathrm{p}}} .
$$

By Lemma 4.18, since $j \mid: F_{\mathrm{c}}^{\prime} \rightarrow X_{\mathrm{c}}$ is an immersed surface representing $\beta$ in $H_{1}\left(K_{\mathrm{c}}\right)$,

$$
x\left(F_{\mathrm{c}}^{\prime}\right) \geq\|\beta\|_{K_{\mathrm{c}}}=m\left\|\gamma_{\mathrm{c}}\right\|_{K_{\mathrm{c}}} .
$$

Combining the estimates above, $x(F) \geq m\left(\|\gamma\|_{\hat{K}_{\mathrm{p}}}+\left\|\gamma_{\mathrm{c}}\right\|_{K_{\mathrm{c}}}\right)$, thus,

$$
\|\gamma\|_{\hat{K}_{\mathrm{p}}}+\left\|\gamma_{\mathrm{c}}\right\|_{K_{\mathrm{c}}} \leq \frac{x(F)}{m}<\|\gamma\|_{K}+\epsilon .
$$

We conclude that $\|\gamma\|_{\hat{K}_{\mathrm{p}}}+\left\|\gamma_{\mathrm{c}}\right\|_{K_{\mathrm{c}}} \leq\|\gamma\|_{K}$, as $\epsilon>0$ is arbitrary.

\section{Braid satellites}

In this section, we introduce and study braid satellites.

5A. Braid patterns. We shall fix a product structure on $T^{2} \cong S^{1} \times S^{1}$ throughout this section. By a braid we shall mean an embedding $b: S^{1} \hookrightarrow S^{1} \times D^{2}$, whose image is a simple closed loop transverse to the fiber disks. We usually write $k_{b}$ for the classical knot in $S^{3}$ associated to $b$, namely, the "satellite" knot with the trivial companion and the pattern $b$.

There is a family of patterns arising from braids: 
Definition 5.1. Let $b: S^{1} \hookrightarrow S^{1} \times D^{2}$ be a braid. Define the standard braid pattern $P_{b}$ associated to $b$ as $P_{b}=\operatorname{id}_{S^{1}} \times b: S^{1} \times S^{1} \hookrightarrow \Theta^{4}$, where $\Theta^{4}=S^{1} \times S^{1} \times D^{2}$ is the thickened torus. The standard braid torus $K_{b}$ associated to $b$ is defined as the desatellite $T_{\mathrm{std}} \cdot P_{b}$.

Remark 5.2. The standard braid torus $K_{b}$ is sometimes called the spun $T^{2}$-knot obtained from the associated knot $k_{b}$. In [Hirose 1993], the extendable subgroup $\mathscr{E}_{K_{b}}$ has been explicitly computed.

Lemma 5.3. If $b: S^{1} \hookrightarrow S^{1} \times D^{2}$ is a braid with winding number $w(b)$, then $w\left(P_{b}\right)=w(b)$. In particular, $w\left(P_{b}\right) \neq 0$.

Proof. This follows immediately from the construction and the definition of winding numbers.

Proposition 5.4. Suppose $b$ is a braid whose associated knot $k_{b}$ is nontrivial. Then

$$
\left\|\mathrm{pt} \times S^{1}\right\|_{K_{b}}=2 g\left(k_{b}\right)-1 \text { and }\left\|S^{1} \times \mathrm{pt}\right\|_{K_{b}}=0,
$$

where $g\left(k_{b}\right)$ denotes the genus of $k_{b}$.

Proof. For simplicity, we write $K_{b}$ and $k_{b}$ as $K$ and $k$, respectively.

To see $\left\|\mathrm{pt} \times S^{1}\right\|_{K} \geq 2 g(k)-1$, the idea is to construct a map between the complements $f: X_{K} \rightarrow M_{k}$, where $X_{K}=S^{4}-K$ and $M_{k}=S^{3}-k$. Let $Y \subset X_{K}$ be the image of the complement $\Theta^{4}-P_{b}$, and $N \subset M_{k}$ be the image of the complement $S^{1} \times D^{2}-b$. There is a natural projection map $f \mid: Y \cong S^{1} \times N \rightarrow N$. As $M_{k}-N$ is homeomorphic to the solid torus, which is an Eilenberg-MacLane space $K(\mathbb{Z}, 1)$, it is not hard to see that $f \mid$ extends as a map $f: X_{K} \rightarrow M_{k}$.

Provided this, for any properly immersed compact orientable surface $j: F \leftrightarrow X_{K}$ whose boundary represents $m[c]$, the norm of $[f \circ j(F)]$ is bounded below by the singular Thurston norm of $k$. As the singular Thurston norm equals the Thurston norm (see [Gabai 1983]), which further equals $2 g(k)-1$ for nontrivial knots, we obtain $\left\|\mathrm{pt} \times S^{1}\right\|_{K} \geq 2 g(k)-1$.

To see $\left\|\mathrm{pt} \times S^{1}\right\|_{K}=2 g(k)-1$, it suffices to find a surface realizing the norm. In fact, one may first take an inclusion $\iota: \Theta^{4} \rightarrow S^{1} \times D^{3}$, where $\iota=\operatorname{id}_{S^{1}} \times \iota^{\prime}$ and where $\iota^{\prime}: S^{1} \times D^{2} \rightarrow D^{3}$ is a standard unknotted embedding, that is, whose core is unknotted in $D^{3}$ and $S^{1} \times \mathrm{pt} \subset S^{1} \times \partial D^{2}$ is the longitude. Then $K_{b}$ factorizes through a smooth embedding $S^{1} \times D^{3} \hookrightarrow S^{4}$ (unique up to isotopy) via $\iota \circ P_{b}$. This allows us to put a minimal genus Seifert surface of $k$ into $X_{K}$ so that it is bounded by the slope $\mathrm{pt} \times S^{1}$. Thus $\left\|\mathrm{pt} \times S^{1}\right\|_{K}=2 g(k)-1$.

From the factorization above, we may also free-homotope $\left(\iota \circ P_{b}\right)\left(S^{1} \times \mathrm{pt}\right)$ to $S^{1} \times\left\{\mathrm{pt}^{\prime}\right\}$, where $\mathrm{pt}^{\prime}$ is a point on $\partial D^{3}$, via an annulus $S^{1} \times\left[\mathrm{pt}, \mathrm{pt}^{\prime}\right]$ where [pt, $\left.\mathrm{pt}^{\prime}\right]$ is an arc whose interior lies in $D^{3}-k$. As $S^{1} \times\left\{\mathrm{pt}^{\prime}\right\}$ bounds a disk outside the image of $S^{1} \times D^{3}$ in $S^{4}$, we see that $\left\|S^{1} \times \mathrm{pt}\right\|_{K}=0$. 
5B. Braid satellites. As an application of the Schubert inequality for seminorms, we estimate $\|\cdot\|_{K}$ for braid satellites of braid tori. We need the following notation.

Definition 5.5. Let $K: T^{2} \hookrightarrow S^{4}$ be a knotted torus in $S^{4}$, and $\tau: T^{2} \rightarrow T^{2}$ be an automorphism of $T^{2}$. We define the $\tau$-twist $K^{\tau}$ of $K$ to be the knotted torus $K \circ \tau: T^{2} \hookrightarrow S^{4}$.

It follows immediately that the seminorm changes under a twist according to the formula $\|\gamma\|_{K^{\tau}}=\|\tau(\gamma)\|_{K}$.

Fix a product structure $T^{2} \cong S^{1} \times S^{1}$ as before. We denote the basis vectors [ $\left.S^{1} \times \mathrm{pt}\right]$ and $\left[\mathrm{pt} \times S^{1}\right]$ on $H_{1}\left(T^{2} ; \mathbb{R}\right)$ as $\xi, \eta$, respectively. A braid satellite is known as some knotted torus of the form $K_{b}^{\tau} \cdot P_{b^{\prime}}$, where $b, b^{\prime}$ are braids with nontrivial associated knots, and $\tau \in \operatorname{Mod}\left(T^{2}\right)$. It is said to be a plumbing braid satellite if $\tau(\xi)=\eta$ and $\tau(\eta)=-\xi$.

Proposition 5.6. Suppose $b, b^{\prime}$ are braids with nontrivial associated knots, and $\tau$ is an automorphism of $T^{2}$. Let $K$ be the satellite knotted torus $K_{b}^{\tau} \cdot P_{b^{\prime}}$. Then for any $\gamma=x \xi+y \eta$ in $H_{1}\left(T^{2} ; \mathbb{R}\right)$,

$$
\|\gamma\|_{K} \geq\left(2 g^{\prime}-1\right) \cdot|y|+(2 g-1) \cdot\left|r x+s w^{\prime} y\right| .
$$

Here $g, g^{\prime}>0$ are the genera of the associated knots of $b, b^{\prime}$, respectively, and $w^{\prime}$ is the winding number of $b^{\prime}$, and $r, s$ are the intersection numbers $\xi \cdot \tau(\xi), \xi \cdot \tau(\eta)$, respectively. Moreover, the equality is achieved if $K_{b}^{\tau} \cdot P_{b^{\prime}}$ is a plumbing braid satellite.

We remark that one should not expect the seminorm lower bound be realized in general. For instance, in the extremal case when $\tau$ is the identity, $\pi_{1}(K)$ is exactly the knot group of the satellite of classical knots $k_{b} \cdot b^{\prime}$, and the lower bound for the longitude slope is given by the classical Schubert inequality, which is not realized in general. However, the plumbing case is a little special. It provides examples of slopes on which the seminorm is not realized by the singular genus. In fact, when $c \subset K$ is a slope representing $x \xi+y \eta \in H_{1}\left(T^{2}\right)$, where $x, y$ are coprime odd integers, the formula yields that $\|c\|_{K}$ is an even number, so the integer $g_{K}^{\star}(c)$ can never be $\left(\|c\|_{K}+1\right) / 2$. We shall give some estimate of the singular genus and the genus for plumbing braid satellites in Section 5C.

The corollary below follows immediately from Proposition 5.6 and Lemma 4.6:

Corollary 5.7. With the notation of Proposition 5.6, if $\tau$ is an automorphism of $T^{2}$ not fixing $\xi$ up to sign, then the stable extendable subgroup $\mathscr{E}_{K}^{\mathrm{s}}$ of $\operatorname{Mod}\left(T^{2}\right)$ with respect to $K$, and hence the extendable subgroup $\mathscr{E}_{K}$, is finite.

In the rest of this subsection, we prove Proposition 5.6. 
Lemma 5.8. With the notation of Proposition 5.6,

$$
\|\gamma\|_{K} \geq\left(2 g^{\prime}-1\right) \cdot|y|+(2 g-1) \cdot\left|r x+s w^{\prime} y\right| .
$$

Proof. By Lemma 5.3 and Theorem 4.9, $\|\gamma\|_{K} \geq\|\gamma\|_{K_{b^{\prime}}}+\left\|\tau\left(\gamma_{c}\right)\right\|_{K_{b}}$. Note that we are writing $\gamma_{\mathrm{c}}$ with respect to $K_{b} \cdot P_{b^{\prime}}$, so the second term equals the corresponding term in Theorem 4.9 with respect to the twisted satellite $K_{b}^{\tau} \cdot P_{b^{\prime}}$ via an obvious transformation. By Proposition 5.4, $\|\gamma\|_{K_{b^{\prime}}}=\left(2 g^{\prime}-1\right) \cdot|y|$. As $b^{\prime}$ is a braid, $P_{b^{\prime}}: T^{2} \rightarrow \Theta^{4} \simeq T^{2}$ implies $\gamma_{c}=x \xi+w^{\prime} y \eta$. Write $\tau$ as

$$
\left(\begin{array}{ll}
p & q \\
r & s
\end{array}\right)
$$

in $\operatorname{SL}(2, \mathbb{Z})$ under the given basis $\xi, \eta$. Note it agrees with the notation $r, s$ in the statement. Then it is easy to compute $\tau\left(\gamma_{\mathrm{c}}\right)=\left(p x+q w^{\prime} y\right) \xi+\left(r x+s w^{\prime} y\right) \eta$. By Proposition 5.4 again, $\left\|\tau\left(\gamma_{c}\right)\right\|_{K_{b}}=(2 g-1) \cdot\left|r x+s w^{\prime} y\right|$. Combining these calculations, we obtain the estimate as desired.

Lemma 5.9. With the notation of Proposition 5.6, if $K$ is a plumbing braid satellite,

$$
\|\gamma\|_{K} \leq\left(2 g^{\prime}-1\right) \cdot|y|+(2 g-1) \cdot|x| .
$$

Proof. Because $\|\cdot\|_{K}$ is a seminorm (Lemma 4.3), it suffices to prove $\|\xi\|_{K} \leq 2 g-1$ and $\|\eta\|_{K} \leq 2 g^{\prime}-1$. The complement $X_{K}$ is the union of the companion piece $X_{K_{b}}=S^{4}-K_{b}$ and the pattern piece $Y=\Theta^{4}-P_{b^{\prime}}$. Note that $\pi_{1}\left(X_{K_{b}}\right)=\pi_{1}\left(M_{k_{b}}\right)$ where $M_{k_{b}}=S^{3}-k_{b}$ is the knot complement, and $\pi_{1}(Y)=\mathbb{Z} \times \pi_{1}\left(R_{b^{\prime}}\right)$ where $R_{b^{\prime}}=S^{1} \times D^{2}-b^{\prime}$ is the braid complement. From the construction it is clear that $\pi_{1}(Y) \rightarrow \pi_{1}\left(X_{K}\right)$ factors through the desatellite on the first factor, namely, $\mathbb{Z} \times \pi_{1}\left(M_{k_{b^{\prime}}}\right)$, so the commutator length of $\eta$ in $\pi_{1}\left(X_{K}\right)$ is at most that of $\eta$ in $\pi_{1}\left(M_{k_{b^{\prime}}}\right)$, which is $2 g^{\prime}$. Moreover, the slope $\xi \in \partial X_{K}$ can be free-homotoped to a slope $\xi_{\mathrm{c}}$ on $\partial X_{K_{b}}$ since it is a fiber of $Y=S^{1} \times R_{b^{\prime}}$, and by the construction, it is clear that $\xi_{\mathrm{c}}$ represents the longitude slope of $\pi_{1}\left(\partial M_{k_{b}}\right)$ in $\pi_{1}\left(M_{k_{b}}\right) \cong \pi_{1}\left(X_{K_{b}}\right)$, so the commutator length of $\xi$ in $\pi_{1}\left(X_{K}\right)$ is at most that of $\xi_{\mathrm{c}}$ in $\pi_{1}\left(M_{k_{b}}\right)$, which is $2 g$. This proves the lemma because the commutator length equals the singular genus $g_{K}^{\star}$, which gives upper bounds for the seminorm $\|\cdot\|_{K}$ on slopes (Remark 3.3 and Lemma 4.6).

Now Proposition 5.6 follows from Lemmas 5.8, 5.9.

Remark 5.10. For plumbing braid satellites, since the norm is given by

$$
\|\gamma\|_{K}=\left(2 g^{\prime}-1\right)|y|+(2 g-1)|x|,
$$

the unit ball of the norm of plumbing satellite is the rhombus on the plane with the vertices $( \pm 1 /(2 g-1), 0)$ and $\left(0, \pm 1 /\left(2 g^{\prime}-1\right)\right)$. 
5C. On genera of plumbing braid satellites. In this subsection, we estimate the singular genera and the genera of slopes for plumbing braid satellites. While we obtain a pretty nice estimate for the singular genera, with the error at most one, we are not sure how close our genera upper bound is to being the best possible.

Proposition 5.11. Suppose $b, b^{\prime}$ are braids with nontrivial associated knots, and $K$ is the plumbing braid satellite $K_{b}^{\tau} \cdot P_{b^{\prime}}$. Then for every slope $c \subset K$, we have:

(1) The singular genus satisfies

$$
\frac{\|c\|_{K}+1}{2} \leq g_{K}^{\star}(c) \leq \frac{\|c\|_{K}+3}{2} .
$$

In particular, if c represents $x \xi+y \eta$ with both $x$ and $y$ odd, then

$$
g_{K}^{\star}(c)=\frac{\|c\|_{K}}{2}+1 .
$$

(2) If $c$ represents $x \xi+y \eta$ in $H_{1}\left(T^{2}\right)$, where $x$, $y$ are coprime integers, then the genus satisfies

$$
g_{K}(c) \leq g \cdot|x|+g^{\prime} \cdot|y|+\frac{(|x|-1)(|y|-1)}{2},
$$

where $g, g^{\prime}>0$ denote the genera of the associated knots $k_{b}, k_{b^{\prime}}$ in $S^{3}$, respectively.

We prove Proposition 5.11 in the rest of this subsection. We shall rewrite the slopes $S^{1} \times \mathrm{pt}$, pt $\times S^{1} \subset T^{2}$ as $c_{\xi}, c_{\eta}$, respectively.

We need the notion of Euler number to state the next lemma. Let $Y$ be a simply connected, closed oriented 4-manifold, and let $K: T^{2} \hookrightarrow Y$ be a null-homologous knotted torus embedded in $Y$. Let $X=Y-K$ be the compact exterior of the knotted torus. For any locally flat, properly embedded compact oriented surface with connected boundary, $F \hookrightarrow X$, such that $\partial F$ is mapped homeomorphically onto a slope $c \times$ pt of $K \times \mathrm{pt}$ (which exists by Lemma 3.1), we may take a parallel copy $c \times \mathrm{pt}^{\prime} \subset K \times \mathrm{pt}^{\prime}$ of the slope, and perturb $F$ to be another locally flat, properly embedded copy $F^{\prime} \hookrightarrow X$ bounded by $c \times \mathrm{pt}^{\prime}$, so that $F, F^{\prime}$ are in general position. The algebraic sum of the intersections between $F$ and $F^{\prime}$ gives rise to an integer

$$
e(F ; K) \in \mathbb{Z},
$$

which is known as the Euler number of the normal framing of $F$ induced from $K$. In fact, one can check that $e(F ; K)$ only depends on the class $[F] \in H_{2}(X, K \times \mathrm{pt})$. If $Y$ is orientable but has no preferable choice of orientation, we ambiguously speak of the Euler number up to sign.

Lemma 5.12. There exist two disjoint, properly embedded, orientable compact surfaces $E, E^{\prime} \hookrightarrow X_{K}$, bounded by the slopes $c_{\xi} \times p, c_{\eta} \times p^{\prime}$ in two parallel copies 
of the knotted torus $K \times p, K \times p^{\prime} \subset \partial X$, respectively. Moreover, the genera of $E, E^{\prime}$ are $g, g^{\prime}$, respectively, and the Euler number of the normal framing is $e(E ; K)=e\left(E^{\prime} ; K\right)=0$.

Proof. Regarding $K$ as $T_{\text {std }} \cdot P_{b}^{\tau} \cdot P_{b^{\prime}}$, there is a natural decomposition

$$
X_{K}=X_{0} \cup Y \cup Y^{\prime},
$$

where $X_{0}$ is the compact complement of the unknotted torus $T_{\text {std }}$ in $S^{4}$, and $Y, Y^{\prime}$ are the exteriors of $P_{b}, P_{b^{\prime}}$ in the thickened torus $\Theta^{4}$, respectively. Moreover, $Y$ and $Y^{\prime}$ have natural product structures $c_{\eta} \times R_{b}$ and $c_{\xi} \times R_{b^{\prime}}$, respectively, where $R_{b}$ and $R_{b^{\prime}}$ denote the exteriors of the braids $b$ and $b^{\prime}$, respectively, in the solid torus $S^{1} \times D^{2}$. As before, $\partial Y$ and $\partial Y^{\prime}$ each have two components: $\partial Y$ has $\partial_{\mathrm{c}} Y$ and $\partial_{\mathrm{s}} Y$, $\partial Y^{\prime}$ has $\partial_{\mathrm{c}} Y^{\prime}$ and $\partial_{\mathrm{s}} Y^{\prime}$. Thus $\partial X_{0}$ is glued to $\partial_{\mathrm{c}} Y$, and $\partial_{\mathrm{s}} Y$ is glued to $\partial_{\mathrm{c}} Y^{\prime}$, and $\partial_{\mathrm{s}} Y^{\prime}$ is exactly $\partial X_{K}$.

The knot complement $M_{k_{b}}=S^{3}-k_{b}$ is the union of $R_{b}$ with a solid torus $S^{1} \times D^{2}$. From classical knot theory, there is a genus $g$ Seifert surface $S$ of $k_{b}$ properly embedded in $M_{k_{b}}=S^{3}-k_{b}$, and one can arrange $S$ so that it intersects $S^{1} \times D^{2}$ in a finite collection of $n \geq w$ disjoint parallel fiber disks. Thus $S_{b}=S \cap R_{b}$ is a connected properly embedded orientable compact surface, so that $\partial S_{b}$ has one component on $\partial_{\mathrm{s}} R_{b}$ parallel to the longitude $s$, and $n$ components $c_{1}, \ldots, c_{n}$ on $\partial_{\mathrm{c}} R_{b}$ parallel to pt $\times \partial D^{2}$. Similarly, take a connected subsurface $S_{b^{\prime}} \subset R_{b^{\prime}}$ with $n^{\prime}$ boundary components $c_{1}^{\prime}, \ldots, c_{n^{\prime}}^{\prime}$ on the companion boundary, and one boundary component $s^{\prime}$ on the satellite boundary.

Construct a properly embedded compact annulus $E_{Y^{\prime}}$ in $Y^{\prime}=c_{\xi} \times R_{b^{\prime}}$ by taking the product of $c_{\xi}$ with some arc $\alpha \subset R_{b^{\prime}}-S_{b^{\prime}}$, so that the two endpoints lie on $\partial_{\mathrm{c}} R_{b^{\prime}}$ and $\partial_{\mathrm{s}} R_{b^{\prime}}$, respectively. Construct a properly embedded compact surface $E_{Y^{\prime}}^{\prime} \subset Y^{\prime}$ by taking the product of $S_{b^{\prime}}$ with some point in $c_{\xi}$. Similarly, construct a properly embedded compact surface $E_{Y}$ in $Y=c_{\eta} \times R_{b}$ by taking a product of $S_{b}$ with some point in $c_{\eta}$, and construct a union of $n^{\prime}$ annuli $E_{Y}^{\prime}$ by taking the product of $c_{\eta}$ with $n^{\prime}$ disjoint $\operatorname{arcs} \alpha_{1}^{\prime}, \ldots, \alpha_{n^{\prime}}^{\prime}$ in $R_{b}-S_{b}$, each of whose endpoints lie on $\partial_{\mathrm{c}} R_{b}$ and $\partial_{\mathrm{s}} R_{b}$, respectively. Under the gluing, we obtain two disjoint properly embedded surfaces $E_{Y} \cup E_{Y^{\prime}}$ and $E_{Y}^{\prime} \cup E_{Y^{\prime}}^{\prime}$ in $Y \cup Y^{\prime}$, whose boundaries on $\partial_{\mathrm{s}} Y^{\prime}=\partial X_{K} \cong K \times S^{1}$ are $c_{\xi} \times \mathrm{pt}$ and $c_{\eta} \times \mathrm{pt}$, respectively. Moreover, it is clear that $\partial\left(E_{Y} \cup E_{Y^{\prime}}\right)$ has $n$ other boundary components on $\partial_{\mathrm{c}} Y=\partial X_{0} \cong T_{\text {std }} \times S^{1}$ parallel to $c_{\eta} \times$ pt, and $\partial\left(E_{Y}^{\prime} \cup E_{Y^{\prime}}^{\prime}\right)$ has $n^{\prime}$ other boundary components on $\partial_{\mathrm{c}} Y$ parallel to $c_{\xi} \times \mathrm{pt}$.

It is not hard to see that one can cap off these other boundary components with disjoint properly embedded disks in $X_{0}$. In fact, we may regard $T_{\text {std }}: T^{2} \hookrightarrow S^{4}$ as the composition

$$
T^{2} \cong c_{\xi} \times c_{\eta} \hookrightarrow c_{\xi} \times D^{3} \hookrightarrow S^{4},
$$


where $c_{\eta}$ is a trivial knot in $D^{3}$. Thus the components of $\partial\left(E_{Y}^{\prime} \cup E_{Y^{\prime}}^{\prime}\right)$ that lie on $\partial X_{0}$ can be capped off in $c_{\xi} \times D^{3}$ disjointly. Moreover, the components of $\partial\left(E_{Y} \cup E_{Y^{\prime}}\right)$ lying on $\partial X_{0}$ can be isotoped to the boundary of $c_{\xi} \times D^{3}$, so that they are all $c_{\xi}$-fibers. Because $S^{4}-c_{\xi} \times D^{3}$ is homeomorphic to $D^{2} \times S^{2}$, we may further cap off these fibers in the complement of $c_{\xi} \times D^{3}$ in $S^{4}$.

It is straightforward to check that capping off $E_{Y} \cup E_{Y^{\prime}}$ and $E_{Y}^{\prime} \cup E_{Y^{\prime}}^{\prime}$ yields the surfaces $E$ and $E^{\prime}$, as desired. Note that $e(E ; K)$ vanishes because we can perturb the construction above to obtain a surface disjoint from $E$ bounding a slope parallel to $c_{\xi} \times \mathrm{pt}$ in $K \times \mathrm{pt}$. For the same reason, $e\left(E^{\prime} ; K\right)=0$ as well.

Proof of Proposition 5.11. (1) It suffices to show the upper bound. By Lemma 5.12, there are properly embedded surfaces $E, E^{\prime}$ in $X_{K}$ bounded by $c_{\xi} \times \mathrm{pt}, c_{\eta} \times \mathrm{pt}$, respectively, and the complexity of $E$ and $E^{\prime}$ realizes $\left\|c_{\xi}\right\|_{K}$ and $\left\|c_{\eta}\right\|_{K}$, respectively (Proposition 5.6). Suppose $c \subset K$ is a slope representing $x \xi+y \eta$. By the main theorem of [Massey 1974], there exists an $|x|$-sheet connected covering space $\tilde{E}$ of $E$, which has exactly one boundary component if $x$ is odd, or two boundary components if $x$ is even. By the same method, there is also $\tilde{E}^{\prime}$, which is connected $|y|$-sheet covering $E^{\prime}$ with one or two boundary components. Since $x$ and $y$ are coprime, at most one of them is even, so $\tilde{E} \cup \tilde{E}^{\prime}$ have at most three components. Then there are immersions of these surfaces into $X_{K}$, and by homotoping the image of their boundaries to $K \times \mathrm{pt}$ and taking the band sum to make them connected, we obtain an immersed subsurface $F \rightarrow X_{K}$ bounding the slope $c$. Since we need to add up to two bands to make the boundary of $F$ connected, this yields

$$
2 g_{K}^{\star}(c)-1 \leq-\chi(F) \leq(-\chi(E)) \cdot|x|+\left(-\chi\left(E^{\prime}\right)\right) \cdot|y|+2=\|c\|_{K}+2 .
$$

Note that the last equality follows from Proposition 5.6 as we assumed $K$ is the plumbing braid satellite. This proves the first statement. The "in particular" part is also clear because when $x, y$ are both odd, $\|c\|_{K}$ is an even number by the formula, so $\left(\|c\|_{K} / 2\right)+1$ is the only integer satisfying our estimation.

(2) In this case, we take $|x|$ copies of the embedded surface $E$, and $|y|$ copies of the embedded surface $E^{\prime}$, in $X_{K}$. Because the Euler numbers of the normal framing are zero for $E$ and $E^{\prime}$, we may assume these copies to be disjoint. Isotope their boundaries to $K \times \mathrm{pt}$ in $\partial X_{K}$; we see $|x|$ slopes parallel to $c_{\xi}$, and $|y|$ slopes parallel to $c_{\eta}$. As there are $|x y|$ intersection points, we take $|x y|$ band sums to obtain a properly embedded surface $F \hookrightarrow X_{K}$ bounding the slope $c$. There are $|x|+|y|-1$ bands that contribute to making the boundary of $F$ connected, and each of the other $|x y|-|x|-|y|+1$ bands contributes one half to the genus of $F$. This implies

$$
g_{K}(c) \leq g(F)=g \cdot|x|+g^{\prime} \cdot|y|+\frac{(|x|-1)(|y|-1)}{2},
$$

as desired. 


\section{Miscellaneous examples}

In this section, we exhibit examples to show difference between concepts introduced in this note.

6A. Slopes with vanishing seminorm but positive singular genus. Note that we have already seen slopes whose singular genus do not realize nonvanishing seminorm in plumbing braid satellites; see Proposition 5.6. There are also examples where the seminorm vanishes on some slope with positive singular genus, as follows. Our construction is based on the existence of incompressible knotted Klein bottles.

Denote the Klein bottle as $\Phi^{2}$. A knotted Klein bottle in $S^{4}$ is a locally flat embedding $K: \Phi^{2} \hookrightarrow S^{4}$. We usually denote its image also as $K$, and the exterior $X_{K}=S^{4}-K$ is obtained by removing an open regular neighborhood of $K$ from $S^{4}$ as before in the knotted torus case. We say a knotted Klein bottle $K$ is incompressible if the inclusion $\partial X_{K} \subset X_{K}$ induces an injective homomorphism between the fundamental groups. There exist incompressible Klein bottles in $S^{4}$; see [Kamada 1990, Lemma 4].

Incompressible knotted Klein bottles give rise to examples of slopes on knotted tori which have vanishing seminorm but positive singular genus.

Specifically, let $K: \Phi^{2} \hookrightarrow S^{4}$ be an incompressible knotted Klein bottle. Suppose $\kappa: T^{2} \rightarrow \Phi^{2}$ is a two-fold covering of the Klein bottle $\Phi^{2}$. Perturbing $K \circ \kappa: T^{2} \rightarrow S^{4}$ in the normal direction of $K$ gives rise to a knotted torus $\tilde{K}: T^{2} \hookrightarrow S^{4}$.

Lemma 6.1. With the notation above, $\tilde{K}$ has a slope $c$ such that $\|c\|_{\tilde{K}}=0$, but $g_{\tilde{K}}^{\star}(c)>0$.

Proof. Let $\alpha \subset \Phi^{2}$ be an essential simple closed curve on $K$ so that $\kappa^{-1}(\alpha)$ has two components $c, c^{\prime} \subset T^{2}$. Then $c, c^{\prime}$ are parallel on $T^{2}$. We choose orientations on $c, c^{\prime}$ so that they are parallel as oriented curves. Let $\mathcal{N}(K)$ be a compact regular neighborhood of $K$ so that $Y=\mathcal{N}(K)-\tilde{K}$ is a pair-of-pants bundle over $K$. Then $c$ is freely homotopic to the orientation-reversal of $c^{\prime}$ within $Y$. This implies that $2[c \times \mathrm{pt}] \in H_{1}\left(X_{\tilde{K}}\right)$ is represented by a properly immersed annulus $A \rightarrow X_{\tilde{K}}$ whose boundary with the induced orientation equals $c \cup c^{\prime}$. Therefore, $\|c\|_{K}$ equals zero. However, note that $X_{\tilde{K}}=X_{K} \cup Y$, glued along $\partial X_{K}=\partial \mathcal{N}(K)$. Since $K$ is incompressible, $\partial X_{K}$ is $\pi_{1}$-injective in $X_{K}$. It is also clear that both components of $\partial Y$ are $\pi_{1}$-injective in $Y$. It follows that $\pi_{1}(Y)$ injects into $\pi_{1}\left(X_{\tilde{K}}\right)$, and also that $\pi_{1}\left(\partial X_{\tilde{K}}\right)$ injects into $\pi_{1}\left(X_{\tilde{K}}\right)$. Therefore, the slope $c \times$ pt in $\partial X_{\tilde{K}} \cong \tilde{K} \times S^{1}$ is homotopically nontrivial in $\pi_{1}\left(X_{\tilde{K}}\right)$, so $g_{\tilde{K}}^{\star}(c)$ cannot be zero.

6B. Stably extendable but not extendable automorphisms. It is clear that the stable extendable subgroup $\mathscr{E}_{K}^{\mathrm{s}}$ contains the extendable subgroup $\mathscr{E}_{K}$ for any knotted torus $K: T^{2} \hookrightarrow S^{4}$. They are in general not equal. In fact, we show that the Dehn 
twist along a slope with vanishing singular genus is stably extendable (Lemma 6.2). In particular, it follows that for any unknotted embedded torus $K$, the stable extendable subgroup $\mathscr{E}_{K}^{\mathrm{s}}$ equals $\operatorname{Mod}\left(T^{2}\right)$. However, in this case, the extendable subgroup $\mathscr{E}_{K}$ is a proper subgroup of $\operatorname{Mod}\left(T^{2}\right)$ of index three [Ding et al. 2012; Montesinos 1983]. Thus there are many automorphisms that are stably extendable but not extendable for the unknotted embedding.

Fix an orientation of the torus $T^{2}$. For any slope $c \subset T^{2}$ on the torus, we denote the (right-hand) Dehn twist along $c$ as $\tau_{c}: T^{2} \rightarrow T^{2}$. More precisely, the induced automorphism on $H_{1}\left(T^{2}\right)$ is given by $\tau_{c *}(\alpha)=\alpha+I([c], \alpha)[c]$ for all $\alpha \in H_{1}\left(T^{2}\right)$, where $I: H_{1}\left(T^{2}\right) \times H_{1}\left(T^{2}\right) \rightarrow \mathbb{Z}$ denotes the intersection form. Note that the expression is independent from the choice of the direction of $c$.

The criterion below is inspired from techniques of Susumu Hirose and Akira Yasuhara. However, the reader should beware that our notion of stabilization in this paper does not change the fundamental group of the complement, so it is slightly different from the definition in [Hirose and Yasuhara 2008].

Lemma 6.2. Let $K: T^{2} \hookrightarrow S^{4}$ be a knotted torus. Suppose $c \subset T^{2}$ is a slope with the singular genus $g_{K}^{\star}(c)=0$. Then the Dehn twist $\tau_{c} \in \operatorname{Mod}\left(T^{2}\right)$ along $c$ belongs to the stable extendable subgroup $\mathscr{E}_{K}^{\mathrm{s}}$.

Proof. The idea of this criterion is that, for a closed simply connected oriented 4-manifold $Y$, to have the Dehn twist $\tau_{c}$ extendable over $Y$ via the $Y$-stabilization $K[Y]: T^{2} \hookrightarrow Y$, we need $c$ to bound a locally flat, properly embedded disk of Euler number \pm 1 in the complement of $K[Y]$ in $Y$. Such a $Y$ can always be chosen to be the connected sum of copies of $\mathbb{C P}^{2}$ or $\overline{\mathbb{C P}}$.

Recall that we introduced the Euler number of a surface bounding a slope in Section 5C before the statement of Lemma 5.12. Suppose $D$ is a locally flat, properly embedded disk in $X=Y-K[Y]$ bounded by a slope $c \times$ pt on $K[Y] \times$ pt $\subset \partial X$ with $e(D ; K[Y])= \pm 1$. We claim in this case the Dehn twist $\tau_{c} \in \operatorname{Mod}\left(T^{2}\right)$ along $c$ can be extended as an orientation-preserving self-homeomorphism of $Y$. In fact, following the arguments in the proof of [Hirose and Yasuhara 2008, Theorem 4.1], we may take the compact normal disk bundle $v_{D}$ of $D$, identified as embedded in $X$ such that $v_{D} \cap(K[Y] \times \mathrm{pt})$ is an interval subbundle of $v_{D}$ over $\partial D$. Then $e(D ; K[Y])= \pm 1$ implies that $v_{D} \cap(K[Y] \times \mathrm{pt})$ is a (positive or negative) Hopf band in the 3-sphere $\partial v_{D}$, whose core is $c \times$ pt. Thus $\tau_{c}$ extends over $Y$ as a self-homeomorphism by [Hirose and Yasuhara 2008, Proposition 2.1].

Now it suffices to find a $Y$ fulfilling the assumption of the claim above. Suppose $c \subset K$ is a slope with the singular genus $g_{K}^{\star}(c)=0$. Then there is a map $j: D^{2} \rightarrow X_{K}$ so that $\partial D^{2}$ is mapped homeomorphically onto $c \times$ pt in $\partial X_{K} \cong K \times S^{1}$. We may also assume $j$ to be an immersion by the general position argument. Blowing up all the double points of $j\left(D^{2}\right)$, we obtain an embedding 


$$
j^{\prime}: D^{2} \hookrightarrow X_{K} \#(\overline{\mathbb{C P}})^{\# r}
$$

for some integer $r \geq 0$. Suppose $e\left(j^{\prime}(D) ; K\left[\left(\overline{\mathbb{C P}^{2}}\right)^{\# r}\right]\right)$ equals $s \in \mathbb{Z}$. If $s>1$, we may further blow up $s-1$ points in $j^{\prime}(D) \subset X_{K} \#\left(\overline{\mathbb{C P}^{2}}\right)^{\# r}$. This gives rise to

$$
j^{\prime \prime}: D^{2} \hookrightarrow X_{K} \#\left(\overline{\mathbb{C} P^{2}}\right)^{\#(r+s-1)}
$$

satisfying the assumption of the claim, so the Dehn twist $\tau_{c}$ is extendable over $X=X_{K} \#\left(\mathbb{C P}^{2}\right)^{\#(r+s-1)}$, or in other words, it is $Y$-stably extendable, where $Y=$ $\left(\overline{\mathbb{C P}^{2}}\right)^{\#(r+s-1)}$. If $s<1$, a similar argument using negative blow-ups shows that $\tau_{c}$ is $Y$-stably extendable, where $Y=\left(\mathbb{C P}^{2}\right)^{\#(1-s)} \#\left(\overline{\mathbb{C P}^{2}}\right)^{\# r}$.

\section{Further questions}

In conclusion, for a knotted torus $K: T^{2} \hookrightarrow S^{4}$, the seminorm and the singular genus of a slope are meaningful numerical invariants which are sometimes possible to control using group theoretic methods. However, the genera of slopes seem to be much harder to compute. It certainly deserves further exploration how to combine the group-theoretic methods with the classical 4-manifold techniques when the fundamental group comes into play.

We propose several further questions about genera, seminorm and extendable subgroups. Suppose $K: T^{2} \hookrightarrow S^{4}$ is a knotted torus.

Question 7.1. When is the unit disk of the seminorm $\|\cdot\|_{K}$ a finite rational polygon, that is, bounded by finitely many segments of rational lines? (See Remark 5.10.)

Question 7.2. If the index of the extendable subgroup $\mathscr{E}_{K}$ in $\operatorname{Mod}\left(T^{2}\right)$ equals three, is $K$ necessarily the knot connected sum of the unknotted torus with a knotted sphere?

Question 7.3. If the stable extendable subgroup $\mathscr{C}_{K}^{\mathrm{s}}$ equals $\operatorname{Mod}\left(T^{2}\right)$, does the singular genus $g_{K}^{\star}$ vanish for every slope?

Question 7.4. If $K$ is incompressible, that is, $\partial X_{K}$ is $\pi_{1}$-injective in the complement $X_{K}$, is the stable extendable subgroup $\mathscr{E}_{K}^{\mathrm{s}}$ finite?

Question 7.5. For plumbing knotted satellites, does the upper bound in Proposition 5.11(2) realize the genus of the slope?

\section{Acknowledgements}

The authors are grateful to Seiichi Kamada for clarifying some points during the development of the paper and for very helpful guidance to the literature. The authors also thank David Gabai, Cameron Gordon, and Charles Livingston for suggestions and comments, and thank the referee for encouraging us to improve the structure of this paper. 


\section{References}

[Agol et al. 2006] I. Agol, J. Hass, and W. Thurston, "The computational complexity of knot genus and spanning area", Trans. Amer. Math. Soc. 358:9 (2006), 3821-3850. MR 2007k:68037 Zbl 1098.57003

[Artin 1925] E. Artin, “Zur Isotopie zweidimensionaler Flächen im R4”, Abh. Math. Semin. Univ. Hamb. 4 (1925), 174-177. JFM 51.0450.02

[Brunner et al. 1982] A. M. Brunner, E. J. Mayland, Jr., and J. Simon, "Knot groups in $S^{4}$ with nontrivial homology”, Pacific J. Math. 103:2 (1982), 315-324. MR 85a:57014 Zbl 0522.57018

[Calegari 2009] D. Calegari, scl, MSJ Memoirs 20, Mathematical Society of Japan, Tokyo, 2009. MR 2011b:57003 Zbl 1187.20035

[Carter and Saito 1998] J. S. Carter and M. Saito, Knotted surfaces and their diagrams, Mathematical Surveys and Monographs 55, American Mathematical Society, Providence, RI, 1998. MR 98m:57027 Zbl 0904.57010

[Carter et al. 2004] S. Carter, S. Kamada, and M. Saito, Surfaces in 4-space, Encyclopaedia of Mathematical Sciences: Low-Dimensional Topology, III 142, Springer, Berlin, 2004. MR 2005e:57065

[Ding et al. 2012] F. Ding, Y. Liu, S. Wang, and J. Yao, "A spin obstruction for codimension-two homeomorphism extension", Math. Res. Lett. 19 (2012), 345-357.

[Fox 1962] R. H. Fox, "A quick trip through knot theory”, pp. 120-167 in Topology of 3-manifolds and related topics (Athens, GA, 1961), Prentice-Hall, Englewood Cliffs, NJ, 1962. MR 25 \#3522 Zbl 06075327

[Freedman and Quinn 1990] M. H. Freedman and F. Quinn, Topology of 4-manifolds, Princeton Mathematical Series 39, Princeton University Press, 1990. MR 94b:57021 Zbl 0705.57001

[Friedl and Vidussi 2012] S. Friedl and S. Vidussi, "The Thurston norm and twisted Alexander polynomials", preprint, 2012. arXiv 1204.6456v2

[Gabai 1983] D. Gabai, "Foliations and the topology of 3-manifolds", J. Differential Geom. 18:3 (1983), 445-503. MR 86a:57009 Zbl 0533.57013

[Gordon 1976] C. M. Gordon, "Knots in the 4-sphere", Comment. Math. Helv. 51:4 (1976), 585-596. MR 55 \#13435 Zbl 0346.55004

[Gordon 1981] C. M. Gordon, "Homology of groups of surfaces in the 4-sphere", Math. Proc. Cambridge Philos. Soc. 89:1 (1981), 113-117. MR 83d:57016 Zbl 0454.57021

[Hillman 1989] J. Hillman, 2-knots and their groups, Australian Mathematical Society Lecture Series 5, Cambridge University Press, 1989. MR 90d:57025 Zbl 0669.57008

[Hirose 1993] S. Hirose, "On diffeomorphisms over $T^{2}$-knots", Proc. Amer. Math. Soc. 119:3 (1993), 1009-1018. MR 93m:57025 Zbl 0806.57011

[Hirose 2002] S. Hirose, "On diffeomorphisms over surfaces trivially embedded in the 4-sphere", Algebr. Geom. Topol. 2 (2002), 791-824. MR 2003f:57042 Zbl 1074.60090

[Hirose and Yasuhara 2008] S. Hirose and A. Yasuhara, "Surfaces in 4-manifolds and their mapping class groups”, Topology 47:1 (2008), 41-50. MR 2009c:57049 Zbl 1197.57019

[Hosokawa and Kawauchi 1979] F. Hosokawa and A. Kawauchi, "Proposals for unknotted surfaces in four-spaces”, Osaka J. Math. 16:1 (1979), 233-248. MR 81c:57018 Zbl 0404.57020

[Kamada 1990] S. Kamada, "Orientable surfaces in the 4-sphere associated with nonorientable knotted surfaces”, Math. Proc. Cambridge Philos. Soc. 108:2 (1990), 299-306. MR 91i:57014 Zbl 0714.57011 
[Kamada 2002] S. Kamada, Braid and knot theory in dimension four, Mathematical Surveys and Monographs 95, American Mathematical Society, Providence, RI, 2002. MR 2003d:57050 Zbl 0993.57012

[Kanenobu 1983] T. Kanenobu, "Groups of higher-dimensional satellite knots", J. Pure Appl. Algebra 28:2 (1983), 179-188. MR 85d:57018 Zbl 0516.57011

[Kawauchi 1996] A. Kawauchi, A survey of knot theory, Birkhäuser, Basel, 1996. MR 97k:57011 Zbl 0861.57001

[Kawauchi et al. 1982] A. Kawauchi, T. Shibuya, and S. Suzuki, "Descriptions on surfaces in fourspace, I: Normal forms", Math. Sem. Notes Kobe Univ. 10:1 (1982), 75-125. MR 84d:57017 Zbl 0506.57014

[Levine 1978] J. Levine, "Some results on higher dimensional knot groups", pp. 243-273 in Knot theory (Plans-sur-Bex, 1977), edited by J.-C. Hausmann, Lecture Notes in Mathematics 685, Springer, Berlin, 1978. MR 80j:57021 Zbl 0404.57019

[Litherland 1979] R. A. Litherland, "Deforming twist-spun knots", Trans. Amer. Math. Soc. 250 (1979), 311-331. MR 80i:57015 Zbl 0413.57015

[Litherland 1981] R. A. Litherland, "The second homology of the group of a knotted surface", Quart. J. Math. Oxford Ser. (2) 32:128 (1981), 425-434. MR 83a:57024 Zbl 0506.57013

[Livingston 1985] C. Livingston, "Stably irreducible surfaces in $S^{4}$ ", Pacific J. Math. 116:1 (1985), 77-84. MR 86g:57024 Zbl 0559.57018

[Livingston 1988] C. Livingston, "Indecomposable surfaces in 4-space”, Pacific J. Math. 132:2 (1988), 371-378. MR 89g:57030 Zbl 0665.57024

[Maeda 1977] T. Maeda, "On the groups with Wirtinger presentations", Math. Sem. Notes Kobe Univ. 5:3 (1977), 347-358. MR 57 \#13966 Zbl 0376.55004

[Massey 1974] W. S. Massey, "Finite covering spaces of 2-manifolds with boundary", Duke Math. J. 41 (1974), 875-887. MR 50 \#8493 Zbl 0291.57001

[Montesinos 1983] J. M. Montesinos, "On twins in the four-sphere, I", Quart. J. Math. Oxford Ser.

(2) 34:134 (1983), 171-199. MR 86i:57025a Zbl 0522.57019

[Ozsváth and Szabó 2004] P. Ozsváth and Z. Szabó, "Holomorphic disks and genus bounds", Geom. Topol. 8 (2004), 311-334. MR 2004m:57024 Zbl 1056.57020

[Schubert 1953] H. Schubert, "Knoten und Vollringe", Acta Math. 90 (1953), 131-286. MR 17,291d Zbl 0051.40403

[Schubert 1961] H. Schubert, "Bestimmung der Primfaktorzerlegung von Verkettungen”, Math. Z. 76 (1961), 116-148. MR 25 \#4519b Zbl 0097.16302

[Seifert 1935] H. Seifert, “Über das Geschlecht von Knoten”, Math. Ann. 110:1 (1935), 571-592. MR 1512955 Zbl 0010.13303

[Shinohara 1971] Y. Shinohara, "Higher dimensional knots in tubes", Trans. Amer. Math. Soc. 161 (1971), 35-49. MR 44 \#4763 Zbl 0203.25903

[Skopenkov 2008] A. B. Skopenkov, "Embedding and knotting of manifolds in Euclidean spaces", pp. 248-342 in Surveys in contemporary mathematics, edited by N. Young and Y. Choi, London Math. Soc. Lecture Note Ser. 347, Cambridge University Press, 2008. MR 2009e:57040 Zbl 1154.57019 [Thurston 1986] W. P. Thurston, A norm for the homology of 3-manifolds, Mem. Amer. Math. Soc. 59, American Mathematical Society, Providence, RI, 1986. MR 88h:57014 Zbl 0585.57006

[Wu 1958] W.-t. Wu, "On the isotopy of $C^{r}$-manifolds of dimension $n$ in Euclidean $(2 n+1)$-space", Sci. Record (N.S.) 2 (1958), 271-275. MR 21 \#3027 Zbl 0119.38606 
[Yajima 1962] T. Yajima, "On the fundamental groups of knotted 2-manifolds in the 4-space", $J$. Math. Osaka City Univ. 13 (1962), 63-71. MR 27 \#1941 Zbl 0118.39301

[Zeeman 1965] E. C. Zeeman, "Twisting spun knots”, Trans. Amer. Math. Soc. 115 (1965), 471-495. MR 33 \#3290 Zbl 0134.42902

Received December 7, 2011. Revised September 9, 2012.

YI LIU

DEPARTMENT OF MATHEMATICS

CALIFORNia Institute of TECHNOLOGY

374 Sloan Hall, 1200 East CALIFORnia BLVd

PASADENA, CA 91125

UNITED STATES

yliumath@caltech.edu

YI NI

DePartment of Mathematics

California Institute of TECHNOLOGY

251 Sloan Hall, 1200 East California Blvd

PASADENA, CA 91125

UNITED STATES

yini@caltech.edu

\section{HONGBIN SUN}

DEPARTMENT OF MATHEMATICS

PRINCETON UNIVERSITY

Fine HALL, ROOM 304

WASHINGTON ROAD

PRINCETON, NJ 08544

UNITED STATES

hongbins@princeton.edu

SHICHENG WANG

SCHOOL OF Mathematical SCIENCES

PEKING UNIVERSITY

BEIJING, 100871

CHINA

wangsc@math.pku.edu.cn 


\title{
PACIFIC JOURNAL OF MATHEMATICS
}

\author{
msp.org/pjm
}

Founded in 1951 by E. F. Beckenbach (1906-1982) and F. Wolf (1904-1989)

\section{EDITORS}

V. S. Varadarajan (Managing Editor)

Department of Mathematics

University of California

Los Angeles, CA 90095-1555

pacific@math.ucla.edu

Paul Balmer

Department of Mathematics

University of California

Los Angeles, CA 90095-1555

balmer@math.ucla.edu

Daryl Cooper

Department of Mathematics

University of California

Santa Barbara, CA 93106-3080 cooper@math.ucsb.edu

Jiang-Hua $\mathrm{Lu}$

Department of Mathematics

The University of Hong Kong

Pokfulam Rd., Hong Kong jhlu@maths.hku.hk
Don Blasius

Department of Mathematics University of California

Los Angeles, CA 90095-1555

blasius@math.ucla.edu

Robert Finn

Department of Mathematics Stanford University

Stanford, CA 94305-2125

finn@math.stanford.edu

Sorin Popa

Department of Mathematics

University of California

Los Angeles, CA 90095-1555

popa@math.ucla.edu

Paul Yang

Department of Mathematics

Princeton University

Princeton NJ 08544-1000

yang@math.princeton.edu

\section{PRODUCTION}

Silvio Levy, Scientific Editor, production@msp.org

\section{SUPPORTING INSTITUTIONS}

ACADEMIA SINICA, TAIPEI

CALIFORNIA INST. OF TECHNOLOGY

INST. DE MATEMÁTICA PURA E APLICADA

KEIO UNIVERSITY

MATH. SCIENCES RESEARCH INSTITUTE

NEW MEXICO STATE UNIV.

OREGON STATE UNIV.

\author{
STANFORD UNIVERSITY \\ UNIV. OF BRITISH COLUMBIA \\ UNIV. OF CALIFORNIA, BERKELEY \\ UNIV. OF CALIFORNIA, DAVIS \\ UNIV. OF CALIFORNIA, LOS ANGELES \\ UNIV. OF CALIFORNIA, RIVERSIDE \\ UNIV. OF CALIFORNIA, SAN DIEGO \\ UNIV. OF CALIF., SANTA BARBARA
}

\author{
Vyjayanthi Chari \\ Department of Mathematics \\ University of California \\ Riverside, CA 92521-0135 \\ chari@math.ucr.edu \\ Kefeng Liu \\ Department of Mathematics \\ University of California \\ Los Angeles, CA 90095-1555 \\ liu@math.ucla.edu \\ Jie Qing \\ Department of Mathematics \\ University of California \\ Santa Cruz, CA 95064 \\ qing@cats.ucsc.edu
}

These supporting institutions contribute to the cost of publication of this Journal, but they are not owners or publishers and have no responsibility for its contents or policies.

See inside back cover or msp.org/pjm for submission instructions.

The subscription price for 2013 is US \$400/year for the electronic version, and \$485/year for print and electronic.

Subscriptions, requests for back issues and changes of subscribers address should be sent to Pacific Journal of Mathematics, P.O. Box 4163, Berkeley, CA 94704-0163, U.S.A. The Pacific Journal of Mathematics is indexed by Mathematical Reviews, Zentralblatt MATH, PASCAL CNRS Index, Referativnyi Zhurnal, Current Mathematical Publications and the Science Citation Index.

The Pacific Journal of Mathematics (ISSN 0030-8730) at the University of California, c/o Department of Mathematics, 798 Evans Hall \#3840, Berkeley, CA 94720-3840, is published monthly except July and August. Periodical rate postage paid at Berkeley, CA 94704, and additional mailing offices. POSTMASTER: send address changes to Pacific Journal of Mathematics, P.O. Box 4163, Berkeley, CA 94704-0163.

PJM peer review and production are managed by EditFLOW ${ }^{\circledR}$ from Mathematical Sciences Publishers.

PUBLISHED BY

mathematical sciences publishers

nonprofit scientific publishing

http://msp.org/

(C) 2013 Mathematical Sciences Publishers 


\section{PACIFIC JOURNAL OF MATHEMATICS}

Volume $261 \quad$ No. $1 \quad$ January 2013

Hierarchies and compatibility on Courant algebroids

Paulo Antunes, Camille Laurent-GengouX and

JoANA M. NunES DA COSTA

A new characterization of complete linear Weingarten hypersurfaces in real 33 space forms

Cícero P. Aquino, Henrique F. DE Lima and

MARCO A. L. VELÁSQUEZ

Calogero-Moser versus Kazhdan-Lusztig cells

CÉDRIC BONNAFÉ and RAPHAËL ROUQUIER

Coarse median spaces and groups

BRIAN H. BOWDITCH

Geometrization of continuous characters of $\mathbb{Z}_{p}^{\times}$

CLIFTON CUNNINGHAM and MASOUd KAMGARPOUR

A note on Lagrangian cobordisms between Legendrian submanifolds of $\mathbb{R}^{2 n+1}$

\section{ROMAN GOLOVKO}

On slope genera of knotted tori in 4-space

Yi LIU, Yi Ni, HoNGBIN SUN and SHICHENG WANG

Formal groups of elliptic curves with potential good supersingular reduction

$$
\text { ÁlVARO LOZANO-ROBLEDO }
$$

Codimension-one foliations calibrated by nondegenerate closed 2-forms

DAVID MARTínez TORRES

The trace of Frobenius of elliptic curves and the $p$-adic gamma function

DERMOT MCCARTHY

$(D N)-(\Omega)$-type conditions for Fréchet operator spaces 